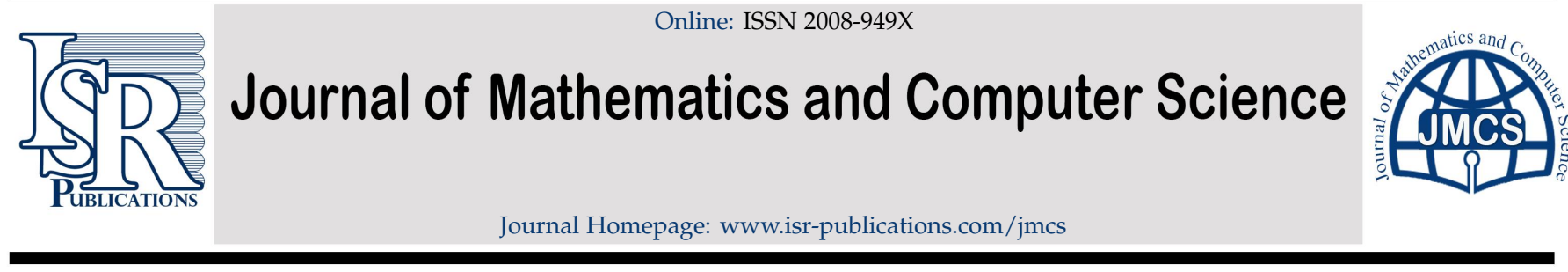

\title{
Fuzzy soft Hilbert spaces
}

\author{
Nashat Faried ${ }^{a}$, Mohamed S.S. Alib, Hanan H. Sakr ${ }^{b, *}$ \\ ${ }^{a}$ Department of Mathematics, Faculty of Science, Ain Shams University, 11566 Cairo, Egypt. \\ ${ }^{b}$ Department of Mathematics, Faculty of Education, Ain Shams University, 11341 Cairo, Egypt.
}

\begin{abstract}
In this work, we define the fuzzy soft Hilbert space $\tilde{\mathrm{H}}$ based on the definition of the fuzzy soft inner product space $(\tilde{\mathrm{U}}, \widetilde{<\cdot \cdot>})$, introduced by Faried et al. [N. Faried, M. S. S. Ali, H. H. Sakr, Appl. Math. Inf. Sci., 14 (2020), 709-720], in terms of the fuzzy soft vector $\tilde{v}_{f_{G(e)}}$. Moreover, we show that $\mathbb{C}^{n}(A), \mathbb{R}^{n}(A)$ and $\ell_{2}(A)$ are suitable examples of fuzzy soft Hilbert spaces. In addition, it is proved that the fuzzy soft orthogonal complement of any non-empty fuzzy soft subset of $\tilde{H}$ is a fuzzy soft closed fuzzy soft subspace of $\tilde{H}$ and we study some of the fuzzy soft Hilbert spaces properties and some of the fuzzy soft inner product spaces properties. Furthermore, we introduce the definition of the fuzzy soft orthogonal family and the fuzzy soft orthonormal family and introduce examples satisfying them. Moreover, we present the fuzzy soft Bessel's inequality and the fuzzy soft Parseval's formula in this generalized setting.
\end{abstract}

Keywords: Fuzzy set, fuzzy soft Hilbert space, fuzzy soft inner product space, fuzzy soft linear space, fuzzy soft set, soft set. 2020 MSC: 46B99, 03E72, 46S40.

(C)2021 All rights reserved.

\section{Introduction}

In the real world, complexity generally arises from uncertainty in the form of ambiguity. So, we always have many complicated problems in areas like economics, engineering, medicine, environmental science, sociology, business management and many other sciences. We can't successfully use classical mathematical methods to overcome difficulties of uncertainties in those problems.

In 1965, Zadeh [20] proposed an extension of the set theory which is the theory of fuzzy sets to deal with uncertainty. Just as a crisp set on a universal set $X$ is defined by its characteristic function from $X$ to $\{0,1\}$, a fuzzy set on a domain $X$ is defined by its membership (characteristic) function from $X$ to $[0,1]$. Fuzzy set theory is a very useful mathematical tool to handle uncertainty, but this single value (membership degree) combines the evidence for element's belonging and the evidence against element's belonging without indicating how much there is of each, i.e., the single number tells us nothing about its accuracy.

In 1999, Molodtsov [15] introduced an extension of the set theory namely soft set theory to overcome uncertainties and solve complicated problems which can't be dealt with by classical methods in many

\footnotetext{
*Corresponding author

Email address: hananhassan@edu . asu .edu.eg (Hanan H. Sakr)

doi: $10.22436 /$ jmcs.022.02.06

Received: 2020-01-26 Revised: 2020-04-10 Accepted: 2020-06-15
} 
areas such as Riemann integration, measure theory, environmental science, decision making, game theory, physics, engineering, computer science, medicine, economics and many other fields. The soft set is a mathematical tool for modeling uncertainty by associating a set with a set of parameters, i.e., it is a parameterized family of subsets of the universal set. After that, many researchers introduced new extended concepts based on soft sets, gave examples for them and studied their properties like soft point [1], soft metric spaces [6], soft normed spaces [19], soft inner product spaces [5] and soft Hilbert spaces [18], etc. But almost all the time, although this progress, in real-life problems and situations, we still have inexact information about our considered objects. So, to improve those two concepts; fuzzy set and soft set, Maji et al. [13] combined them together in one concept and called this new concept fuzzy soft set. This new concept widened the approach of the soft set from crisp (ordinary) cases to fuzzy cases which is more general than any other.

In recent years, many researchers applied this notion and gave some concepts and applications such as fuzzy soft point [16], fuzzy soft real number [8], fuzzy soft metric spaces [2] and fuzzy soft normed spaces [3]. In addition, many researchers presented some applications of soft set theory and fuzzy soft set theory in different areas (see [9, 12,17]). Recently, Faried et al. [7] progressed on these stated previous studies by introducing the fuzzy soft inner product on fuzzy soft vector spaces, studying its properties and giving some related results like fuzzy soft Cauchy-Schwartz inequality and fuzzy soft orthogonality.

In this paper, we define the fuzzy soft Hilbert space $\tilde{H}$, study its properties and introduce many more related results. The rest of the paper is organized as the following. Section 2 introduces the basic concepts and definitions for the fuzzy set, the soft set and the fuzzy soft set. In addition, it presents fuzzy soft metric space, fuzzy soft normed space and fuzzy soft inner product space, and their properties. Section 3 studies the fuzzy soft Hilbert spaces $\tilde{H}$ in terms of the fuzzy soft vector $\tilde{v}_{f_{\mathrm{G}(e)}}$. In addition, in Section 3 , it is shown that $\mathbb{C}^{\mathfrak{n}}(A), \mathbb{R}^{\mathfrak{n}}(A)$ and $\ell_{2}(A)$ are examples of fuzzy soft Hilbert spaces and we define the fuzzy soft orthogonal family and the fuzzy soft orthonormal family. Moreover, in Section 3, some properties of the fuzzy soft Hilbert spaces and the fuzzy soft inner product spaces are established. Furthermore, in Section 3, fuzzy soft Bessel's inequality and fuzzy soft Parseval's formula are investigated. Section 4 provides conclusions and open questions for further investigation.

\section{Preliminaries}

The aim of this section is to list some notations, definitions and preliminaries of fuzzy set, soft set and fuzzy soft set which shall be needed in the following discussion. In addition, it presents the fuzzy soft point definition including our present modification. Furthermore, it introduces the definitions of fuzzy soft metric space, fuzzy soft normed space and fuzzy soft inner product space, and their properties that have been studied before and can be used in the new results.

Definition 2.1 (Fuzzy set, [20]). Let $U$ be a universal set. A fuzzy set (class) $X$ over $U$ is a set characterized by a function $f_{X}: U \rightarrow[0,1]$. $f_{X}$ is called the membership, characteristic or indicator function of the fuzzy set $X$ and the value $f_{X}(u)$ is called the grade of membership of $u \in U$ in $X$.

Definition 2.2 (Soft set, $[14,15])$. Let $U$ be a universal set (space of points or objects), $E$ be a set of parameters and $A \subseteq E$. The power set of $U$ is defined by $P(U)=2^{U}$. A pair $(F, A)$ is called a soft set over $U$ and is defined as a set of ordered pairs $F_{A}=\left\{\left(e, F_{A}(e)\right): e \in A, F_{A}(e) \in P(U)\right\}$, where $F$ is a mapping given by $F: A \rightarrow P(U)$. $A$ is called the support of $F_{A}$ and we have $F_{A}(e) \neq \phi$ for all $e \in A$ and $F_{A}(e)=\phi$ for all $e \notin A$.

Example 2.1. Consider a soft set $(F, A)$ which describes "attractiveness of markets" under the consideration of a decision maker to purchase. Suppose that there are five markets to be considered in the universal set $U$, denoted by $U=\left\{m_{1}, m_{2}, m_{3}, m_{4}, m_{5}\right\}$ and $A=\left\{a_{1}, a_{2}, a_{3}\right\}$, where $a_{i}(i=1,2,3)$ stands for the parameters in a word of "luxurious", "expensive", and "in a suitable location", respectively. Thus, to define a soft set means to represent luxurious markets, expensive markets and so on. We can write the 
soft set $(F, A)$ over $U$ by the relation $(F, A)=\left\{\left(a_{1},\left\{m_{2}, m_{4}\right\}\right),\left(a_{2},\left\{m_{5}\right\}\right),\left(a_{3},\left\{m_{1}, m_{3}, m_{4}\right\}\right)\right\}$. This soft set can be represented in Table 1.

Table 1: Representation of the soft set $(F, A)$.

\begin{tabular}{|c|ccc|}
\hline $\mathrm{u}$ & $\mathrm{a}_{1}$ & $\mathrm{a}_{2}$ & $\mathrm{a}_{3}$ \\
\hline $\mathrm{m}_{1}$ & 0 & 0 & 1 \\
$\mathrm{~m}_{2}$ & 1 & 0 & 0 \\
$\mathrm{~m}_{3}$ & 0 & 0 & 1 \\
$\mathrm{~m}_{4}$ & 1 & 0 & 1 \\
$\mathrm{~m}_{5}$ & 0 & 1 & 0 \\
\hline
\end{tabular}

Definition 2.3 (Fuzzy soft set, [13]). Let $U$ be a universal set, $E$ be a set of parameters and $A \subseteq E$. A pair $(G, A)$ is called a fuzzy soft set over $U$, where $G$ is a mapping given by $G: A \rightarrow \mathcal{F}(U)$, where $\mathcal{F}(U)$ is the family of all fuzzy subsets of $U$ (the power set of fuzzy sets on $U$ ) and the fuzzy subset of $U$ is defined as a map from $U$ to $[0,1]$.

Example 2.2. Example (2.1) can be characterized by a membership function instead of 0,1 numbers which associates each element with a real number in the interval $[0,1]$, then we can write the fuzzy soft set $(G, A)$ over $U$ as $(G, A)=\left\{G\left(a_{1}\right)=\left\{\left(m_{1}, 0.1\right),\left(m_{2}, 0.6\right),\left(m_{4}, 0.8\right),\left(m_{5}, 0.3\right)\right\}, G\left(a_{2}\right)=\left\{\left(m_{4}, 0.2\right),\left(m_{5}, 0.8\right)\right\}, G\left(a_{3}\right)=\right.$ $\left.\left\{\left(m_{1}, 1\right),\left(m_{3}, 0.7\right),\left(m_{4}, 0.9\right)\right\}\right\}$. This fuzzy soft set can be represented in Table 2.

Table 2: Representation of the fuzzy soft set $(G, A)$.

\begin{tabular}{|c|ccr|}
\hline $\mathrm{u}$ & $\mathrm{a}_{1}$ & $\mathrm{a}_{2}$ & $\mathrm{a}_{3}$ \\
\hline $\mathrm{m}_{1}$ & 0.1 & 0 & 1 \\
$\mathrm{~m}_{2}$ & 0.6 & 0 & 0 \\
$\mathrm{~m}_{3}$ & 0 & 0 & 0.7 \\
$\mathrm{~m}_{4}$ & 0.8 & 0.2 & 0.9 \\
$\mathrm{~m}_{5}$ & 0.3 & 0.8 & 0 \\
\hline
\end{tabular}

In order to discuss efficiently, we consider only all fuzzy soft sets $(G, A)$ over a universal set $U$ in which all the parameter sets $A$ are the same. The family of all these fuzzy soft sets is denoted by $F S S(U)_{A}=$ $\operatorname{FSS}(\tilde{\mathrm{U}})$.

The following definition and its consequent related definitions take their present formula according to our modification as follows:

Definition 2.4 (Fuzzy soft point, [16]). The fuzzy soft set $(G, A) \in F S S(\tilde{U})$ is called a fuzzy soft point over $\mathrm{U}$, denoted by $\left(u_{f_{\mathrm{G}(e)}}, A\right)$ (briefly denoted by $\left.\tilde{u}_{f_{\mathrm{G}(e)}}\right)$, if for the element $e \in A$ and $u \in U(\alpha \in(0,1]$ is the value of the membership degree),

$$
f_{G(e)}(u)= \begin{cases}\alpha, & \text { if } u=u_{0} \in U \text { and } e=e_{0} \in A \\ 0, & \text { if } u \in U-\left\{u_{0}\right\} \text { or } e \in A-\left\{e_{0}\right\}\end{cases}
$$

The fuzzy soft point can be considered as the quadruple $\left(u_{0}, e_{0}, G, \alpha\right)$.

Example 2.3. Suppose that there are four points in the universal set $U$, i.e., $U=\left\{u^{1}, u^{2}, u^{3}, u^{4}\right\}$ and let $A=\left\{e_{1}, e_{2}, e_{3}, e_{4}, e_{5}\right\} \subseteq E$ be the set of parameters. Then, $\left(u^{2}, e_{5}, G, 0.6\right)=\tilde{u}_{0.6_{G}\left(e_{5}\right)}$ is a fuzzy soft point over U.

Definition 2.5 (The complement of a fuzzy soft point, [11]). The fuzzy soft point $\tilde{u}_{f_{G} c_{(e)}}$ is called the fuzzy soft complement of a fuzzy soft point $\tilde{u}_{f_{G}(e)}$, denoted by $\left(\tilde{u}_{f_{G(e)}}{ }^{C}\right.$, if for the element $e \in A$ and $u \in U$,

$$
f_{G^{C}(e)}(u)= \begin{cases}1-f_{G(e)}(u), & \text { if } u=u_{0} \in U \text { and } e=e_{0} \in A \\ 0, & \text { if } u \in U-\left\{u_{0}\right\} \text { or } e \in A-\left\{e_{0}\right\}\end{cases}
$$


Example 2.4. The complement of the fuzzy soft point stated in Example 2.3 is the fuzzy soft point $\left(u^{2}, e_{5}, G, 0.4\right)=\tilde{u}^{2}{ }_{0.4} G_{\left(e_{5}\right)}$.

The collection of all fuzzy soft points over $U$ is denoted by $\operatorname{FSP}(U)_{A}=\operatorname{FSP}(\tilde{U}) . \mathbb{R}(A)$ denotes the set of all fuzzy soft real numbers and $\mathbb{R}^{+}(A)$ denotes the set of all non-negative fuzzy soft real numbers (such as, $\tilde{r}$, in symbol). $\mathbb{C}(A)$ denotes the set of all fuzzy soft complex numbers (such as, $\tilde{\mathrm{c}}$, in symbol). Note that the fuzzy soft zero vector $\tilde{\theta}=(\tilde{0}, \tilde{0}, \tilde{0}, \tilde{0})$ and the fuzzy soft unit vector $\tilde{j}=(\tilde{1}, \tilde{1}, \tilde{1}, \tilde{1})$.

Definition 2.6 (Fuzzy soft vector space, [3]). Let $U$ be a vector space over a field $\mathbb{K}(\mathbb{K}=\mathbb{R})$ and the parameter set $E$ be the set of all real numbers $\mathbb{R}$ and $A \subseteq E$. The fuzzy soft set $(G, A) \in F S S(\tilde{U})$ is called a fuzzy soft vector over $U$, denoted by $\left(v_{f_{\mathrm{G}(e)}}, A\right)$ (briefly denoted by $\left.\tilde{v}_{f_{\mathrm{G}(e)}}\right)$, if there is exactly one $e \in A$ such that $f_{G(e)}(v)=\alpha$ for some $v \in U$ and $f_{G\left(e^{\prime}\right)}(v)=0$ for all $e^{\prime} \in A-\{e\}(\alpha \in(0,1]$ is the value of the membership degree). The set of all fuzzy soft vectors over $U$ is denoted by FSV $(U)_{A}=F S V(\tilde{U})$. The set $\tilde{V} \tilde{=F S V}(\tilde{U})$ is said to be a fuzzy soft vector space or a fuzzy soft linear space of $U$ over $\mathbb{K}$ if $\tilde{V}(e)$ is a vector subspace of $\mathrm{U}$, for all $e \in A$. $\tilde{V}$ is a fuzzy soft vector space according to the following two operations:

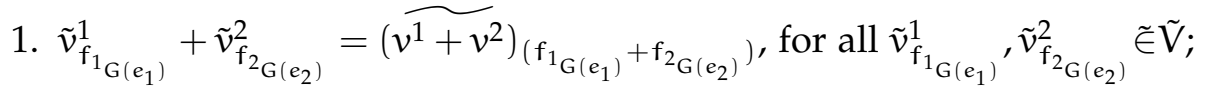

2. $\tilde{r} \cdot \tilde{v}_{f_{G(e)}}=(\widetilde{r})_{f_{G(r e)}}$, for all $\tilde{v}_{f_{G}(e)} \tilde{\in} \tilde{V}$ and for all $\tilde{r} \tilde{\in} \mathbb{R}(A)$.

Example 2.5. Consider the Euclidean $n$-dimensional space $\mathbb{R}^{\mathfrak{n}}$ over $\mathbb{R}$. Let $A=\{1,2,3, \ldots, n\}$ be the set of parameters. Let $\tilde{V}: A \rightarrow \mathcal{F}\left(\mathbb{R}^{n}\right)$ be defined as follows: $\tilde{V}(i)=\left\{\tilde{v}_{f_{n_{G}\left(e_{n}\right)}^{n}}^{n} \tilde{\in} \mathbb{R}^{n}(A) ; i^{\text {th }}\right.$ coordinate of $\tilde{v}_{f_{n_{G}\left(e_{n}\right)}^{n}}^{n}$ is $\tilde{\theta}\}, i=1,2, \ldots, n$. Then $\tilde{V}$ is a fuzzy soft vector space or a fuzzy soft linear space of $\mathbb{R}^{n}$ over $\mathbb{R}$. In addition, Let $\tilde{v}_{f_{n_{G}\left(e_{n}\right)}^{n}}^{n}$ be a fuzzy soft element of $\tilde{V}$ as follows: $\tilde{v}_{f_{n_{G}\left(e_{n}\right)}^{n}}^{n}=\left(\tilde{j}, \tilde{j}, \ldots, \tilde{j}, \tilde{\theta}_{\left(i^{\text {th }}\right)}, \tilde{j}, \ldots, \tilde{j}\right) \tilde{\in} \mathbb{R}^{n}(A)$, $i=1,2, \ldots, n$. Then $\tilde{v}_{f_{n_{G}\left(e_{n}\right)}^{n}}$ is a fuzzy soft vector of $\tilde{V}$.

Definition 2.7 (Fuzzy soft metric space, [2]). A fuzzy soft metric space $(\tilde{U}, \tilde{d})$ is a fuzzy soft set $\tilde{U}$ with a fuzzy soft real-valued function $\tilde{\mathrm{d}}: \operatorname{FSP}(\tilde{\mathrm{U}}) \times \operatorname{FSP}(\tilde{\mathrm{U}}) \rightarrow \mathbb{R}^{+}(A)$ satisfying the fuzzy soft metric conditions as the following:

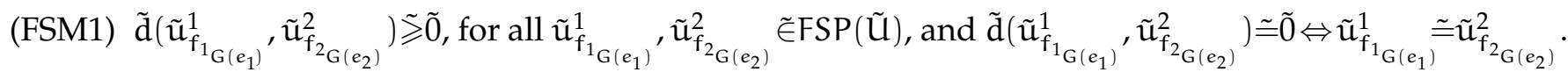

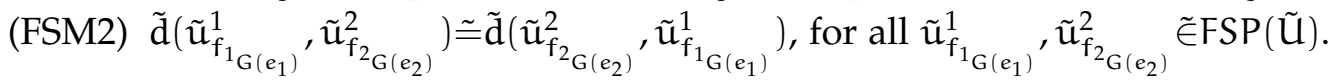

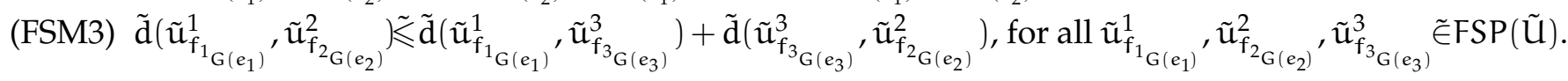

Let $\mathrm{U}$ be a vector space over a field $\mathbb{K}(\mathbb{K}=\mathbb{R})$ and the parameter set $E$ be the set of all real numbers $\mathbb{R}$ and $A \subseteq E$.

Definition 2.8 (Fuzzy soft normed space, [3,4]). Let FSV(Ũ) be a fuzzy soft vector space. Then, a mapping $\widetilde{\|\cdot\|}: \operatorname{FSV}(\tilde{\mathrm{U}}) \rightarrow \mathbb{R}^{+}(\mathrm{A})$ is said to be a fuzzy soft norm on $\operatorname{FSV}(\tilde{\mathrm{U}})$ if $\|\cdot\|$ satisfies the following conditions:

(FSN1) $\left\|\widetilde{\tilde{v}_{\mathrm{f}_{\mathrm{G}(e)}}}\right\| \geqslant \tilde{0}$, for all $\tilde{v}_{\mathrm{f}_{\mathrm{G}(e)}} \tilde{\in} \mathrm{FSV}(\tilde{\mathrm{U}})$, and $\left\|\widetilde{\tilde{v}_{\mathrm{f}_{\mathrm{G}(e)}}}\right\| \tilde{=} \tilde{0} \Leftrightarrow \tilde{v}_{\mathrm{f}_{\mathrm{G}(e)}} \tilde{=} \tilde{\theta}$.

(FSN2) $\left\|\widetilde{\boldsymbol{r} \cdot \tilde{v}_{f_{G(e)}}}\right\| \tilde{=}|\tilde{r}|\left\|\widetilde{\tilde{v}_{f_{G}(e)}}\right\|$, for all $\tilde{v}_{f_{G(e)}} \tilde{\in F S V}(\tilde{\mathrm{U}})$ and for all fuzzy soft scalar $\tilde{r}$.

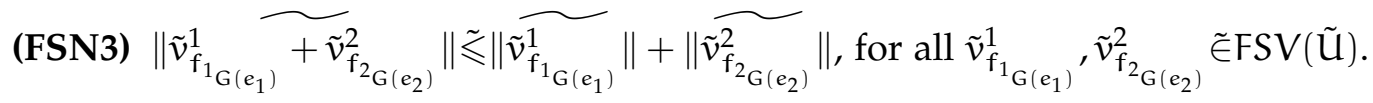

The fuzzy soft vector space FSV $(\tilde{\mathrm{U}})$ with a fuzzy soft norm $\|\cdot\|$ is said to be a fuzzy soft normed linear space and is denoted by $(\tilde{\mathrm{U}}, \widetilde{\|\cdot\|})$. 
Definition 2.9 (Fuzzy soft convergence, [10]). A sequence of fuzzy soft vectors $\left\{\tilde{v}_{f_{n_{G}\left(e_{n}\right)}^{n}}^{n}\right\}$ in a fuzzy soft normed space $(\tilde{\mathrm{U}}, \widetilde{\|\cdot\|})$ is said to be fuzzy soft convergent and converges to $\tilde{v}_{\mathrm{f}_{0_{\mathrm{G}}\left(\mathrm{e}_{0}\right)}}^{0}$, if we have, $\lim _{n \rightarrow \infty}\left\|\tilde{v}_{f_{n_{G}\left(e_{n}\right)}^{n}}-\tilde{v}_{f_{f_{G}\left(e_{0}\right)}^{0}}^{0}\right\| \tilde{=} \tilde{0}$, i.e., $\forall \tilde{\epsilon} \tilde{>} \tilde{0}, \exists n_{0} \in \mathbb{N}$ such that $\left\|\tilde{v}_{f_{n_{G}\left(e_{n}\right)}^{n}}-\tilde{v}_{f_{f_{G}\left(e_{0}\right)}^{0}}^{0}\right\| \tilde{<} \tilde{\epsilon}, \forall n \geqslant n_{0}$. It is denoted by

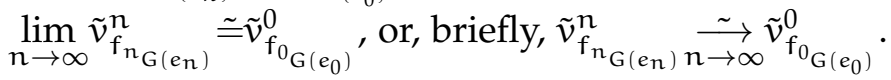

Definition 2.10 (Fuzzy soft Cauchy sequence, [10]). A sequence of fuzzy soft vectors $\left\{\tilde{v}_{\mathbf{f}_{\mathrm{G}_{\mathrm{G}}\left(\mathrm{e}_{\mathfrak{n}}\right)}^{n}}^{\text {n }}\right\}$ in a fuzzy soft normed space $(\tilde{U}, \widetilde{\|\cdot\|})$ is said to be a fuzzy soft Cauchy sequence, if

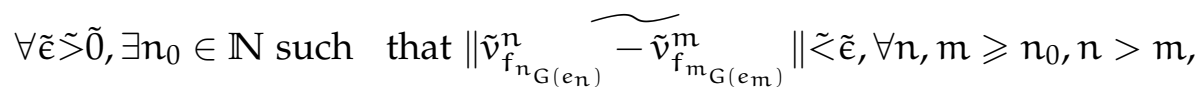

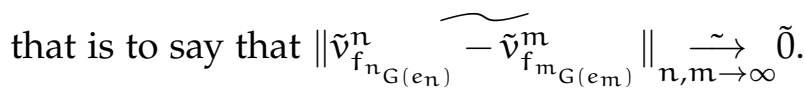

Proposition 2.11 ([10]). Every fuzzy soft convergent sequence is a fuzzy soft Cauchy sequence.

Definition 2.12 (Fuzzy soft completeness, [10]). A fuzzy soft normed space $(\tilde{\mathrm{U}}, \widetilde{\|} \cdot \|)$ is called fuzzy soft complete if every fuzzy soft Cauchy sequence in it is fuzzy soft convergent sequence in it.

Definition 2.13 (Fuzzy soft Banach space, [10]). Every fuzzy soft complete fuzzy soft normed linear space is called a fuzzy soft Banach space.

Theorem 2.14 ([10]). Every fuzzy soft normed linear space is a fuzzy soft metric space with the fuzzy soft metric

$$
\tilde{\mathrm{d}}\left(\tilde{v}_{\mathrm{f}_{1_{\mathrm{G}}\left(e_{1}\right)}}^{1}, \tilde{v}_{\mathrm{f}_{\mathrm{G}_{\mathrm{G}}\left(e_{2}\right)}}^{2}\right) \tilde{=}\left\|\tilde{v}_{\mathrm{f}_{\mathrm{G}_{\mathrm{G}\left(e_{1}\right)}}^{1}} \widetilde{-\tilde{v}_{\mathrm{f}_{\mathrm{G}\left(e_{2}\right)}}^{2}}\right\| \text {, for all } \tilde{v}_{\mathrm{f}_{\mathrm{f}_{\mathrm{G}}\left(e_{1}\right)}}^{1}, \tilde{v}_{\mathrm{f}_{\mathrm{f}_{\mathrm{G}}\left(e_{2}\right)}}^{2} \tilde{\in} \mathrm{FSV}(\tilde{\mathrm{U}}) \text {. }
$$

Definition 2.15 (Fuzzy soft inner product space, [7]). Let FSV(Ũ) be a fuzzy soft vector space. Then, the mapping $\widetilde{\langle\cdot, \cdot\rangle}: \operatorname{FSV}(\tilde{\mathrm{U}}) \times \mathrm{FSV}(\tilde{\mathrm{U}}) \rightarrow \mathbb{C}(A)$ is said to be a complex fuzzy soft inner product (shortly, fuzzy soft inner product) on $\operatorname{FSV}(\tilde{\mathrm{U}})$ if $\widetilde{\mathcal{Z}, \cdot \cdot>}$ satisfies the following axioms:

(FSI1) $<\tilde{v}_{\mathrm{f}_{\mathrm{G}(e)}, \tilde{v}_{\mathrm{f}_{\mathrm{G}(e)}}}>\tilde{\geqslant} \tilde{0}$, for all $\tilde{v}_{\mathrm{f}_{\mathrm{G}(e)}} \tilde{\in} \mathrm{FSV}(\tilde{\mathrm{U}})$, and $<\tilde{v}_{\mathrm{f}_{\mathrm{G}(e)}, \widetilde{v_{\mathrm{f}_{\mathrm{G}(e)}}}}>\tilde{=} \tilde{0} \Leftrightarrow \tilde{v}_{\mathrm{f}_{\mathrm{G}(e)}} \tilde{=} \tilde{\theta}$.

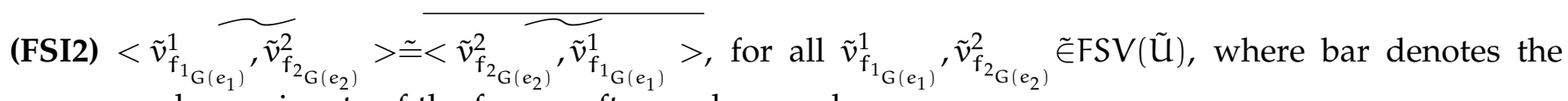
complex conjugate of the fuzzy soft complex number.

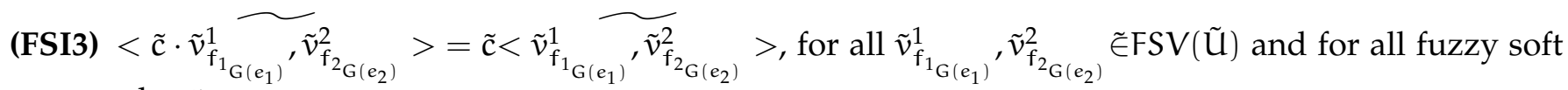
scalar $\tilde{c}$.

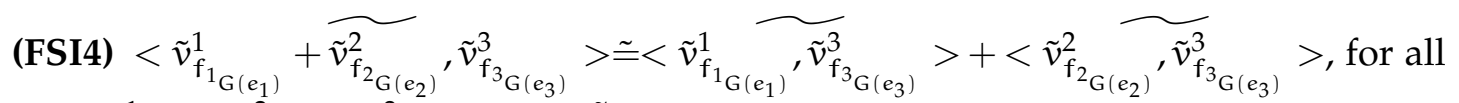
$\tilde{v}_{f_{\mathrm{G}_{\mathrm{G}}\left(e_{1}\right)}}^{1}, \tilde{v}_{\mathrm{f}_{\mathrm{G}_{\mathrm{G}}\left(e_{2}\right)}^{2}}, \tilde{v}_{\mathrm{f}_{3_{\mathrm{G}}\left(e_{3}\right)}^{3}} \tilde{\in} \mathrm{FSV}(\tilde{\mathrm{U}})$.

The fuzzy soft vector space FSV( $\tilde{U})$ with a fuzzy soft inner product $\widetilde{r_{r} \cdot>}$ is said to be a complex fuzzy soft inner product space (shortly, fuzzy soft inner product space) and is denoted by $(\tilde{\mathrm{U}}, \widetilde{<\cdot, \cdot>})$.

Theorem 2.16 ([7]). In fuzzy soft inner product spaces, we have the following:

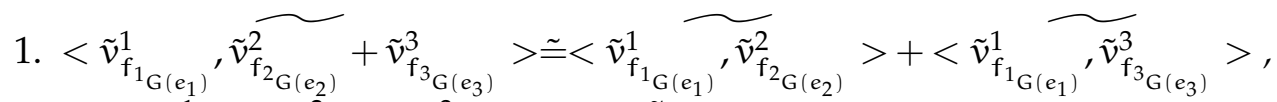
for all $\tilde{v}_{f_{1_{G}\left(e_{1}\right)}^{1}}^{1}, \tilde{v}_{f_{\mathrm{G}_{\mathrm{G}}\left(e_{2}\right)}^{2}}^{2}, \tilde{v}_{\mathrm{f}_{\mathrm{G}_{\mathrm{G}}\left(\mathrm{e}_{3}\right)}^{3}}^{3} \tilde{\mathrm{FSSV}}(\tilde{\mathrm{U}})$. 


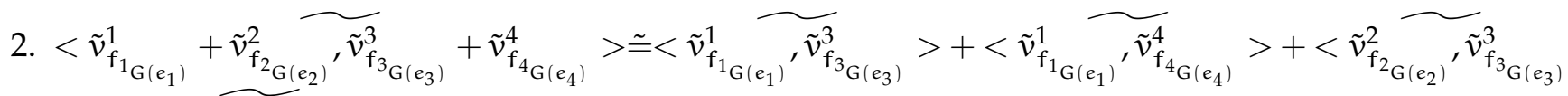

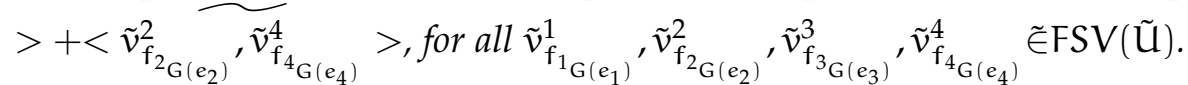

3. For fuzzy soft complex scalars $\tilde{\alpha}_{i}, i: 1, \ldots, n$ and $\tilde{\beta}_{j}, j: 1, \ldots, m$,

$$
<\sum_{i=1}^{n} \tilde{\alpha}_{i} \tilde{v}_{f_{i_{G}\left(e_{i}\right)}^{i}}^{i^{\prime}} \sum_{j=1}^{m} \tilde{\beta}_{j} \tilde{u}_{f_{j_{G}\left(e_{j}\right)}^{j}}^{j}>\tilde{=} \sum_{i=1}^{n} \sum_{j=1}^{m} \tilde{\alpha}_{i} \overline{\tilde{\beta}_{j}}<\tilde{v}_{f_{i_{G}\left(e_{i}\right)}^{i}}^{{ }_{i}, \tilde{u}_{f_{j_{G}\left(e_{j}\right)}^{j}}}>.
$$

4. If for fixed $\tilde{v}_{\mathrm{f}_{\mathrm{G}(e)}} \tilde{\in} \mathrm{FSV}(\tilde{\mathrm{u}})$, we have $<\tilde{v}_{\mathrm{f}_{\mathrm{G}(e)}, \tilde{\mathrm{u}}_{\mathrm{g}_{\mathrm{G}(\mathrm{a})}}}>\tilde{=} \tilde{0}$ for all $\tilde{\mathrm{u}}_{\mathrm{g}_{\mathrm{G}(\mathrm{a})}} \tilde{\in} \mathrm{FSV}(\tilde{\mathrm{u}})$, then, $\tilde{v}_{\mathrm{f}_{\mathrm{G}(e)}} \tilde{=} \tilde{\theta}$.

Definition 2.17 (Real fuzzy soft inner product space, [7]). If the mapping $\widetilde{<\cdot, \cdot>}$ in the above Definition 2.15 is replaced by $\widetilde{<\cdot, \cdot>}: \operatorname{FSV}(\tilde{\mathrm{U}}) \times \operatorname{FSV}(\tilde{\mathrm{U}}) \rightarrow \mathbb{R}(A)$, then it is called a real fuzzy soft inner product space and its conditions (Axioms (FSI1), (FSI3), (FSI4)) are the same, but the condition (FSI2) is replaced by

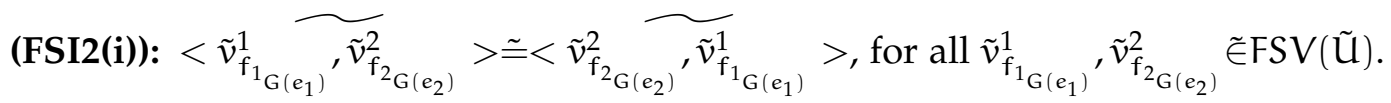

Theorem 2.18 (Fuzzy soft Cauchy-Schwartz inequality, [7]). Let $(\tilde{\mathrm{U}}, \widetilde{<\cdot, \cdot>})$ be a fuzzy soft inner product space, then for all $\tilde{v}_{\mathrm{f}_{\mathrm{G}_{\mathrm{G}}\left(e_{1}\right)}^{1}}, \tilde{v}_{\mathrm{f}_{\mathrm{f}_{\mathrm{G}}\left(\mathrm{e}_{2}\right)}^{2}} \tilde{\mathrm{E}} \mathrm{FSV}(\tilde{\mathrm{U}})$, we have:

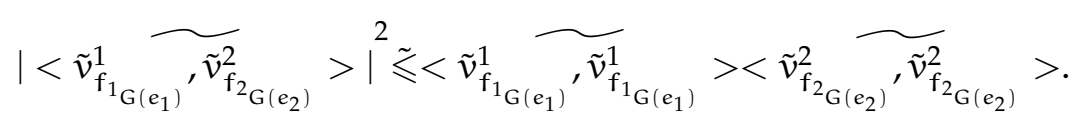

Theorem 2.19 ([7]). A fuzzy soft inner product space $(\tilde{\mathrm{U}}, \widetilde{<\cdot, \cdot>})$ can be considered as a fuzzy soft normed space with the fuzzy soft norm $\left\|\widetilde{\tilde{v}_{f_{\mathrm{G}(e)}}}\right\| \tilde{=} \sqrt{\left\langle\tilde{v}_{\mathrm{f}_{\mathrm{G}(e)}, \tilde{v}_{\mathrm{f}_{\mathrm{G}(e)}}}\right.}$, for all $\tilde{v}_{\mathrm{f}_{\mathrm{G}(e)}} \tilde{\in} \mathrm{FSV}(\tilde{\mathrm{U}})$.

Example 2.6. $\mathbb{C}^{n}(A)$ the fuzzy soft complex Euclidean space (the space of all fuzzy soft $n$-dimensional complex numbers) is a complex fuzzy soft inner product space with the complex fuzzy soft inner product defined as follows:

$$
<\tilde{v}_{f_{G(e)}, \tilde{u}_{g_{G(a)}}}>\cong \sum_{i=1}^{n} \tilde{v}_{f_{i_{G}\left(e_{i}\right)}^{i}} \frac{\tilde{u}_{g_{i_{G}\left(a_{i}\right)}}^{i}}{}
$$

for all $\tilde{v}_{f_{G(e)}}, \tilde{u}_{g_{G(a)}} \tilde{\in} \mathbb{C}^{n}(A)$.

Solution. The proof is straightforward by applying the conditions of the complex fuzzy soft inner product space stated in Definition 2.15.

Example 2.7. $\mathbb{R}^{\mathfrak{n}}(A)$ the fuzzy soft real Euclidean space (the space of all fuzzy soft $n$-dimensional real numbers) is a real fuzzy soft inner product space with the real fuzzy soft inner product defined as follows:

$$
<\tilde{v}_{f_{G(e)}, \tilde{u}_{g_{G(a)}}}>\simeq \sum_{i=1}^{n} \tilde{v}_{f_{i_{G}\left(e_{i}\right)}^{i}} \frac{\tilde{u}_{g_{i_{G}\left(a_{i}\right)}}^{i}}{},
$$

for all $\tilde{v}_{f_{\mathrm{G}(e)}}, \tilde{\mathrm{u}}_{\mathrm{g}_{\mathrm{G}(\mathrm{a})}} \tilde{\in} \mathbb{R}^{\mathrm{n}}(\mathrm{A})$.

Proof. The proof is straightforward by applying the conditions of the real fuzzy soft inner product space stated in Definition 2.17.

Example 2.8. $\ell_{2}(A)$ the space of all fuzzy soft square-summable sequences is a complex fuzzy soft inner product space with the complex fuzzy soft inner product defined as follows:

$$
<\tilde{v}_{f_{G(e)}, \tilde{u}_{g_{G(a)}}}>\simeq \sum_{i=1}^{\infty} \tilde{v}_{f_{i_{G}\left(e_{i}\right)}^{i}} \frac{\tilde{u}_{g_{i_{G}\left(a_{i}\right)}}^{i}}{},
$$

for all $\tilde{v}_{f_{G(e)}}, \tilde{u}_{g_{G(a)}} \tilde{\in} \ell_{2}(A)$. 
Proof. The proof is straightforward by applying the conditions of the complex fuzzy soft inner product space stated in Definition 2.15.

Example 2.9. $\mathcal{C}_{[\tilde{0}, \tilde{1}]}(A)$ the space of all fuzzy soft complex-valued continuous functions on $[\tilde{0}, \tilde{1}]$ is a complex fuzzy soft inner product space with the complex fuzzy soft inner product defined as follows:

$$
<\tilde{\eta}_{\left.f_{G(e)}\right)} \tilde{\varepsilon}_{g_{G(a)}}>\tilde{=} \int_{i=\tilde{0}}^{\tilde{1}} \tilde{\eta}_{f_{i_{G}\left(e_{i}\right)}^{i}}(\tilde{x}) \bar{\xi}_{\tilde{g}_{i_{G}\left(a_{i}\right)}^{i}(\tilde{x})} d \tilde{x},
$$

for all $\tilde{\eta}_{f_{G(e)}}, \tilde{\xi}_{g_{G(a)}} \in \mathcal{C}_{[\tilde{0}, \tilde{1}]}(A)$.

Proof. The proof is straightforward by applying the conditions of the complex fuzzy soft inner product space stated in Definition 2.15.

Theorem 2.20 (Fuzzy soft parallelogram law, [7]). Let $(\tilde{\mathrm{U}}, \widetilde{<\cdot, \cdot>})$ be a fuzzy soft inner product space and let $\tilde{v}_{f_{1_{G}\left(e_{1}\right)}}^{1}, \tilde{v}_{f_{2_{G}\left(e_{2}\right)}^{2}}^{2} \tilde{F S V}(\tilde{U})$. Then, the fuzzy soft parallelogram law is hold as follows:

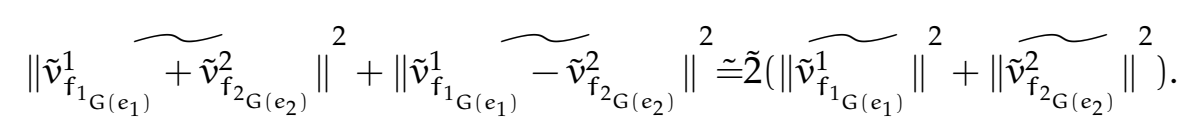

Theorem 2.21 (Fuzzy soft continuity property, [7]). Let $(\tilde{\mathrm{u}}, \widetilde{<\cdot, \cdot>})$ be a fuzzy soft inner product space. If $\lim _{n \rightarrow \infty} \tilde{v}_{e_{n}}^{n} \tilde{=} \tilde{v}_{e_{0}}^{0}$ and $\lim _{n \rightarrow \infty} \tilde{u}_{e_{n}}^{n} \tilde{=} \tilde{u}_{e_{0}}^{0}$, then $\lim _{n \rightarrow \infty}<\widetilde{v_{e_{n}}, \tilde{u}_{e_{n}}^{n}}>\tilde{=}<\widetilde{v_{e_{0}}, \tilde{u}_{e_{0}}^{0}}>$.

Definition 2.22 (Fuzzy soft orthogonality, [7]). Let $(\tilde{\mathrm{U}}, \widetilde{<\cdot, \cdot>})$ be a fuzzy soft inner product space and let $\tilde{v}_{f_{1_{G}\left(e_{1}\right)}}^{1}, \tilde{v}_{f_{f_{G}\left(e_{2}\right)}^{2}}^{2} \tilde{F S V}(\tilde{U})$. Then, $\tilde{v}_{f_{1_{G}\left(e_{1}\right)}}^{1}$ is said to be fuzzy soft orthogonal to $\tilde{v}_{f_{\mathrm{f}_{G}\left(e_{2}\right)}}^{2}$, written $\tilde{v}_{f_{1_{G}\left(e_{1}\right)}}^{1} \tilde{\perp}_{\tilde{v}_{f_{G}\left(e_{2}\right)}}^{2}$, if $<\tilde{v}_{f_{1_{G}\left(e_{1}\right)}^{1}, \tilde{v}_{f_{2_{G}\left(e_{2}\right)}^{2}}^{1}}>\tilde{=0}$.

Theorem 2.23 ([7]). Let $(\tilde{\mathrm{U}}, \widetilde{<\cdot, \cdot>})$ be a fuzzy soft inner product space and let $\tilde{v}_{\mathrm{f}_{\mathrm{G}_{\mathrm{G}}\left(e_{1}\right)}}^{1}, \tilde{v}_{\mathrm{f}_{\mathrm{f}_{\mathrm{G}}\left(\mathrm{e}_{2}\right)}} \tilde{\in} \mathrm{FSV}(\tilde{\mathrm{U}})$. If $\tilde{v}_{f_{1_{G}\left(e_{1}\right)}}^{1} \tilde{\perp} \tilde{v}_{f_{2_{G}\left(e_{2}\right)}^{2}}^{2}$, then

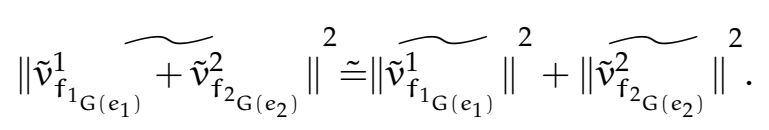

Definition 2.24 (Fuzzy soft orthogonal complement, [7]). Let $(\tilde{\mathrm{U}}, \widetilde{<\cdot, \cdot>})$ be a fuzzy soft inner product space. Let $\tilde{\omega}$ be a non-empty fuzzy soft subset of FSV $(\tilde{U})$. The fuzzy soft set of all fuzzy soft elements (points) $\tilde{v}_{f_{1_{G}\left(e_{1}\right)}^{1}}$ of FSV( $\left.\tilde{U}\right)$ which are fuzzy soft orthogonal to $\tilde{\omega}$ is denoted by $\tilde{\omega}^{\tilde{\perp}}$, and is called the fuzzy soft orthogonal complement of $\tilde{\omega}$. That is to say that

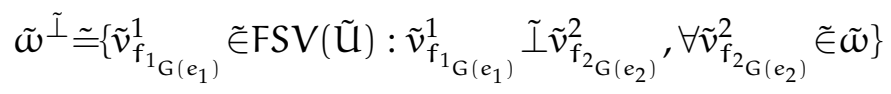

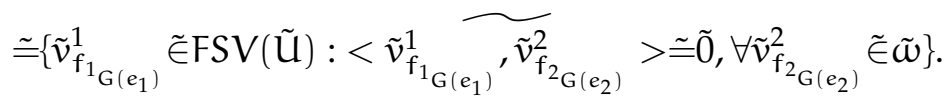

Theorem 2.25 ([7]). For fuzzy soft orthogonal complement, we have:

(1) $\{\tilde{\theta}\}^{\tilde{I}} \tilde{=} \tilde{U}$ and $\tilde{U}^{\tilde{I}} \tilde{=}\{\tilde{\theta}\}$;

(2) $\tilde{\omega} \tilde{\cap} \tilde{\omega} \tilde{\perp} \tilde{=}\{\tilde{\theta}\}$;

(3) $\tilde{\omega}^{\tilde{\perp} \tilde{\perp}} \tilde{=} \tilde{\omega}$;

(4) if $\tilde{\omega}_{1} \widetilde{\subset} \tilde{\omega}_{2}$, then $\tilde{\omega}_{2}^{\tilde{\perp}} \widetilde{\subset} \tilde{\omega}_{1}^{\tilde{\perp}}$. 


\section{Main results}

The aim of this section is to introduce the concept of fuzzy soft Hilbert spaces and to study some properties and examples of fuzzy soft Hilbert spaces. In addition, fuzzy soft orthogonal family and fuzzy soft orthonormal family are established. Finally, fuzzy soft Bessel's inequality and fuzzy soft Parseval's formula are introduced.

Definition 3.1 (Fuzzy soft Hilbert space). Let $(\tilde{\mathrm{U}}, \widetilde{\langle\cdot, \cdot>})$ be a fuzzy soft inner product space. Then, this space, which is fuzzy soft complete in the induced fuzzy soft norm stated in Theorem 2.19, is called a fuzzy soft Hilbert space, denoted by $(\tilde{\mathrm{H}}, \widetilde{\langle\cdot, \cdot\rangle})$ (shortly $\tilde{\mathrm{H}})$. It is clear that every fuzzy soft Hilbert space is a fuzzy soft Banach space.

Example 3.1. The space $\mathbb{C}^{n}(A)$ the fuzzy soft complex Euclidean space (the space of all fuzzy soft ndimensional complex numbers) is a complex fuzzy soft Hilbert space with the complex fuzzy soft inner product defined as follows:

$$
<\tilde{v}_{f_{G(e)}, \tilde{u}_{g_{G(a)}}}>\simeq \sum_{i=1}^{n} \tilde{v}_{f_{i_{G}\left(e_{i}\right)}^{i}} \frac{\tilde{u}_{g_{i_{G}\left(a_{i}\right)}}^{i}}{}
$$

for all $\tilde{v}_{f_{G}(e)}, \tilde{u}_{g_{G(a)}} \tilde{\in} \mathbb{C}^{n}(A)$.

Solution. We have $\mathbb{C}^{\mathfrak{n}}(A)$ is an fuzzy soft inner product space from Example 2.6. Using Theorem 2.19, we obtain:

$$
\left\|\widetilde{\tilde{v}_{f_{G}(e)}}\right\| \tilde{=} \sqrt{<\tilde{v}_{f_{G(e)},} \tilde{v}_{f_{G}(e)}>}=\sum_{i=1}^{n} \tilde{v}_{f_{i_{G}\left(e_{i}\right)}^{i}} \overline{\tilde{v}_{f_{i}\left(e_{i}\right)}^{i}} \tilde{=}\left(\sum_{i=1}^{n}\left|\tilde{v}_{f_{i_{G}\left(e_{i}\right)}^{i}}\right|^{2}\right)^{\frac{1}{2}},
$$

and since $\mathbb{C}^{\mathfrak{n}}(A)$ is fuzzy soft complete in this fuzzy soft norm. Then, $\mathbb{C}^{\mathfrak{n}}(A)$ is an fuzzy soft complete fuzzy soft inner product space, i.e., a fuzzy soft Hilbert space.

Example 3.2. The space $\mathbb{R}^{n}(A)$, the fuzzy soft real Euclidean space (the space of all fuzzy soft $n$ dimensional real numbers) is an fuzzy soft Hilbert space with the real fuzzy soft inner product defined as follows:

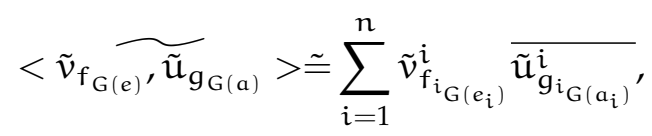

for all $\tilde{v}_{f_{G(e)}}, \tilde{u}_{g_{G(a)}} \tilde{\in} \mathbb{R}^{\mathfrak{n}}(A)$.

Solution. The proof is easy by using Example 2.7 and Theorem 2.19, similarly as Example 3.1.

Example 3.3. The space $\ell_{2}(A)$ the space of all fuzzy soft square-summable sequences is an fuzzy soft Hilbert space with the complex fuzzy soft inner product defined as follows:

$$
<\tilde{v}_{f_{G(e)}, \tilde{u}_{g_{G(a)}}}>\simeq \sum_{i=1}^{\infty} \tilde{v}_{f_{i_{G}\left(e_{i}\right)}^{i}} \frac{\tilde{u}_{g_{i_{G}\left(a_{i}\right)}}^{i}}{}
$$

for all $\tilde{v}_{f_{G(e)}}, \tilde{u}_{g_{G(a)}} \tilde{\in} \ell_{2}(A)$.

Solution. The space $\ell_{2}(A)$ is fuzzy soft complete as an fuzzy soft normed space. But $\ell_{2}(A)$ is an fuzzy soft inner product space from Example 2.8. Then, $\ell_{2}(A)$ is an fuzzy soft Hilbert space.

Theorem 3.2. Let $\tilde{\mathrm{H}}$ be a fuzzy soft Hilbert space. If $\tilde{\boldsymbol{\omega}} \tilde{\mathrm{C}} \tilde{\mathrm{H}}$, then $\tilde{\boldsymbol{\omega}}^{\mathcal{\perp}}$ is a fuzzy soft closed fuzzy soft subspace of $\tilde{H}$. 


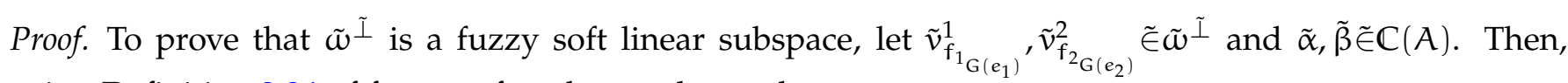
using Definition 2.24 of fuzzy soft orthogonal complement, we get:

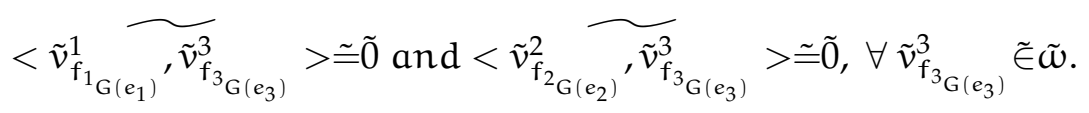

Using the properties which are stated in Theorem 2.16, we obtain:

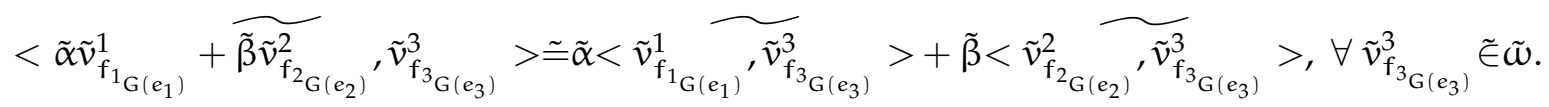

By substituting from (3.1) in (3.2), we have:

$$
<\tilde{\alpha} \tilde{v}_{f_{1_{G}\left(e_{1}\right)}^{1}}^{1}+\widetilde{\tilde{\beta} \tilde{v}_{f_{\mathrm{f}_{G}\left(e_{2}\right)}^{2}}^{2}} \tilde{v}_{\mathrm{f}_{3_{G}\left(e_{3}\right)}}^{3}>\tilde{=} \tilde{\alpha} \times \tilde{0}+\tilde{\beta} \times \tilde{0} \tilde{=} \tilde{0}, \forall \tilde{v}_{\mathrm{f}_{3_{G}\left(e_{3}\right)}}^{3} \tilde{\in} \tilde{\omega} .
$$

Therefore, $\tilde{\alpha} \tilde{v}_{f_{1_{G}\left(e_{1}\right)}^{1}}^{1}+\tilde{\beta} \tilde{v}_{f_{2_{G}\left(e_{2}\right)}^{2}}^{2} \tilde{\epsilon} \tilde{\omega}^{\tilde{\perp}}$, and hence $\tilde{\omega}^{\tilde{\perp}}$ is a fuzzy soft linear subspace of $\tilde{\mathrm{H}}$.

To prove that $\tilde{\omega}^{\tilde{\perp}}$ is a fuzzy soft closed, let $\tilde{v}_{f_{G}(e)} \tilde{\in} \overline{\tilde{\omega}} \tilde{\perp}$, then there exists a fuzzy soft sequence $\tilde{v}_{f_{n_{G}\left(e_{n}\right)}^{n}} \widetilde{\subset} \tilde{\omega}^{\tilde{\perp}}$ and $\lim _{n \rightarrow \infty} \tilde{v}_{f_{n_{G}\left(e_{n}\right)}^{n}} \tilde{=} \tilde{v}_{f_{G}(e)}$. By Theorem 2.9, $\forall \tilde{u}_{g_{G}(a)} \tilde{\in} \tilde{\omega}$, we have:

$$
<\tilde{v}_{f_{G(e)}, \tilde{u}_{g_{G(a)}}}>\simeq \lim _{n \rightarrow \infty}<\tilde{v}_{f_{n_{G}\left(e_{n}\right)}^{n}} \widetilde{u}_{g_{G(a)}}>\simeq \lim _{n \rightarrow \infty} \tilde{0} \tilde{=0} .
$$

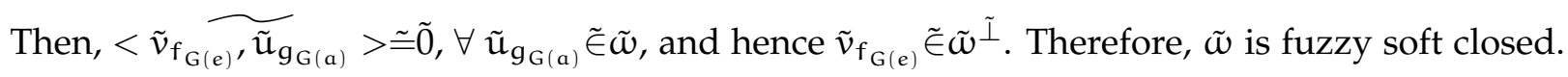

Theorem 3.3. Let $\tilde{\mathrm{H}}$ be a fuzzy soft inner product space and let $\tilde{\omega}_{1}$ and $\tilde{\omega}_{2}$ be two non-empty fuzzy soft linear subspaces of $\tilde{\mathrm{H}}$. Then,

$$
\left(\tilde{\omega}_{1}+\tilde{\omega}_{2}\right)^{\tilde{\perp}} \tilde{=} \tilde{\omega}_{1}^{\tilde{I}} \widetilde{\cap} \tilde{\omega}_{2}^{\tilde{I}} .
$$

Proof. Let $\tilde{v}_{f_{\mathrm{G}(e)}} \tilde{\in} \tilde{\omega}_{1}$, but $\tilde{v}_{f_{\mathrm{G}(e)}} \tilde{=} \tilde{v}_{f_{\mathrm{G}(e)}}+\tilde{\theta}, \tilde{\theta} \tilde{\in} \tilde{\omega}_{2}$, and hence $\tilde{v}_{f_{\mathrm{G}(e)}} \tilde{\in} \tilde{\omega}_{1}+\tilde{\omega}_{2}$. Then, $\tilde{\omega}_{1} \tilde{c}_{\tilde{\omega}_{1}}+\tilde{\omega}_{2}$ and similarly, $\tilde{\omega}_{2} \widetilde{\subset} \tilde{\omega}_{1}+\tilde{\omega}_{2}$. Therefore, using property (4) in Theorem 2.25 , we have $\left(\tilde{\omega}_{1}+\tilde{\omega}_{2}\right)^{\tilde{\perp}} \widetilde{\subset} \tilde{\omega}_{1}^{\tilde{\perp}}$ and $\left(\tilde{\omega}_{1}+\tilde{\omega}_{2}\right) \tilde{\perp} \widetilde{\subset} \tilde{\omega}_{2}^{\tilde{\perp}}$. Hence,

$$
\left(\tilde{\omega}_{1}+\tilde{\omega}_{2}\right)^{\tilde{\perp}} \widetilde{\subset} \tilde{\omega}_{1}^{\tilde{I}} \widetilde{\cap} \tilde{\omega}_{2}^{\tilde{\perp}} .
$$

Conversely, let $\tilde{v}_{f_{\mathrm{G}(e)}} \tilde{\in} \tilde{\omega}_{1}^{\tilde{\perp}} \widetilde{\cap} \tilde{\omega}_{2}^{\tilde{\perp}}$, then $\tilde{v}_{f_{\mathrm{G}(e)}} \tilde{\in} \tilde{\omega}_{1}^{\tilde{\perp}}$ and $\tilde{v}_{f_{\mathrm{G}(e)}} \tilde{\in} \tilde{\omega}_{2}^{\tilde{\perp}}$ and hence using Definition 2.24 of fuzzy soft orthogonal complement, we get:

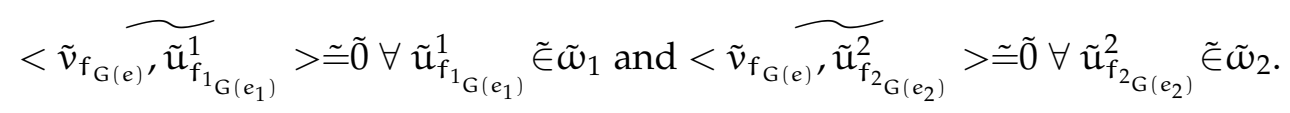

Let $\tilde{\mathfrak{u}}_{\mathrm{g}_{\mathrm{G}(\mathrm{a})}} \tilde{=} \tilde{\mathfrak{u}}_{\mathrm{f}_{1_{\mathrm{G}}\left(e_{1}\right)}^{1}}^{1}+\tilde{\mathfrak{u}}_{\mathrm{f}_{\mathrm{G}_{\mathrm{G}}\left(\mathrm{e}_{2}\right)}^{2}} \tilde{\in} \tilde{\mathfrak{w}}_{1}+\tilde{\mathfrak{w}}_{2}$, then using the properties which are stated in Theorem 2.16, we obtain:

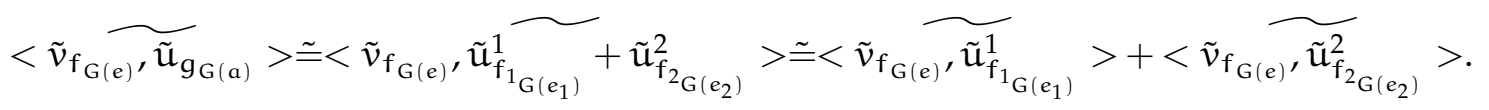

By substituting from (3.5) in (3.6), we have:

$$
<\tilde{v}_{f_{\mathrm{G}(e)}, \tilde{\mathrm{u}}_{\mathrm{g}_{\mathrm{G}(\mathrm{a})}}}>\tilde{=} \tilde{0}+\tilde{0} \tilde{=} \tilde{0} \forall \tilde{\mathrm{u}}_{\mathrm{g}_{\mathrm{G}(\mathrm{a})}} \tilde{\in} \tilde{\mathrm{w}}_{1}+\tilde{\mathrm{w}}_{2} .
$$

Therefore, $\tilde{v}_{f_{\mathrm{G}(e)}} \tilde{\in}\left(\tilde{\omega}_{1}+\tilde{\omega}_{2}\right)^{\tilde{\perp}}$ by Definition 2.24. That is to say that

$$
\tilde{\omega}_{1}^{\tilde{\perp}} \widetilde{\cap} \tilde{\omega}_{2}^{\tilde{\perp}} \widetilde{C}\left(\tilde{\omega}_{1}+\tilde{\omega}_{2}\right)^{\tilde{\perp}} .
$$

Hence, from (3.4) and (3.7), the required Formula (3.3) is true. 
Theorem 3.4. Let $\tilde{\mathrm{H}}$ be a fuzzy soft Hilbert space. If $\tilde{\omega}_{1}$ and $\tilde{\omega}_{2}$ are fuzzy soft closed fuzzy soft subspaces of $\tilde{\mathrm{H}}$ such that $\tilde{\omega}_{1} \tilde{\perp} \tilde{\omega}_{2}$, then $\tilde{\omega}_{1}+\tilde{\omega}_{2}$ is also fuzzy soft closed in $\tilde{\mathrm{H}}$.

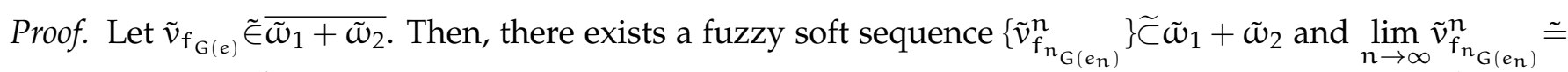
$\tilde{v}_{f_{G(e)}}$. Since $\tilde{\omega}_{1} \tilde{\perp} \tilde{\omega}_{2}$, then any fuzzy soft element $\tilde{u}_{g_{G(a)}} \tilde{\in} \tilde{\omega}_{1}+\tilde{\omega}_{2}$ has a unique form $\tilde{u}_{g_{G(a)}} \tilde{u}_{f_{1_{G}\left(e_{1}\right)}^{1}}+$

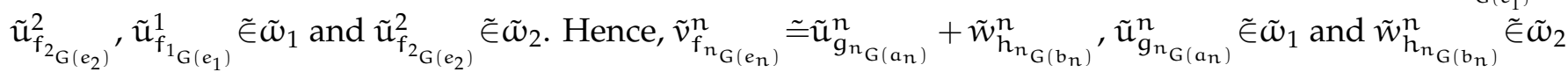

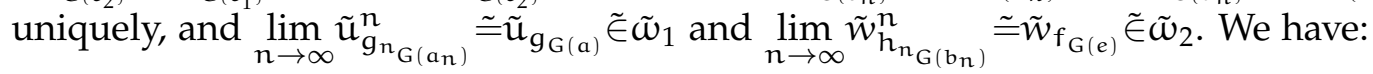

$$
\tilde{v}_{f_{G(e)}} \cong \lim _{n \rightarrow \infty} \tilde{v}_{f_{n_{G}\left(e_{n}\right)}^{n}} \simeq \lim _{n \rightarrow \infty} \tilde{u}_{g_{n_{G}\left(a_{n}\right)}^{n}}^{n}+\lim _{n \rightarrow \infty} \tilde{w}_{h_{n_{G}\left(b_{n}\right)}^{n}} \cong \tilde{u}_{g_{G(a)}}+\tilde{w}_{f_{G(e)}} \tilde{\in} \tilde{\omega}_{1}+\tilde{\omega}_{2} .
$$

Therefore, $\tilde{v}_{f_{\mathrm{G}(e)}} \tilde{\in} \tilde{\omega}_{1}+\tilde{\omega}_{2}$ and hence $\tilde{\omega}_{1}+\tilde{\omega}_{2}$ is fuzzy soft closed.

Theorem 3.5. Let $\tilde{\mathrm{H}}$ be a fuzzy soft Hilbert space and let $\tilde{\omega}$ be a fuzzy soft closed fuzzy soft subspace of $\tilde{\mathrm{H}}$. Then,

$$
\tilde{\mathrm{H}} \tilde{=} \tilde{\mathfrak{\omega}} \oplus \tilde{\omega}^{\tilde{\perp}} \text {. }
$$

Proof. Since $\tilde{\omega}$ and $\tilde{\omega}^{\tilde{\perp}}$ are fuzzy soft orthogonal fuzzy soft subspaces of $\tilde{\mathrm{H}}$, then, by Theorem 3.4 , we have $\tilde{\omega}+\tilde{\omega}^{\tilde{\perp}}$ is fuzzy soft closed fuzzy soft subspace of $\tilde{H}$. Using the Property (3) of Theorem 2.25 which is $\tilde{\omega}^{\tilde{I} \tilde{I}} \tilde{=} \tilde{\omega}$, we get:

$$
\left(\tilde{\omega}+\tilde{\omega}^{\tilde{\perp}}\right)^{\tilde{\perp}} \tilde{=} \tilde{\omega}^{\tilde{\perp}} \tilde{\cap} \tilde{\omega}^{\tilde{\perp} \tilde{\perp}} \tilde{=} \tilde{\omega} \tilde{\perp} \tilde{\cap} \tilde{\omega} \tilde{=}\{\tilde{\theta}\} \tilde{=} \tilde{H}^{\tilde{\perp}},
$$

from the properties (1) and (2) of Theorem 2.25, and hence $\tilde{\mathrm{H}} \tilde{=} \tilde{\omega}+\tilde{\omega}^{\mathcal{I}}$ from the property (4) of Theorem 2.25 and the sum is direct from the property (2) of Theorem 2.25 which is $\tilde{\omega} \widetilde{\cap} \tilde{\omega} \tilde{\perp} \tilde{=}\{\tilde{\theta}\}$. Then, this completes the proof and the required Formula (3.8) is true.

Definition 3.6 (Fuzzy soft orthogonal family). Let $\tilde{H}$ be a fuzzy soft inner product space. A family $\left\{\tilde{v}_{f_{i_{G}\left(e_{i}\right)}^{i}}^{i}\right.$ of fuzzy soft elements of $\tilde{\mathrm{H}}$ is called a fuzzy soft orthogonal family if

$$
\tilde{v}_{f_{i_{G}\left(e_{i}\right)}^{i}}^{i} \tilde{v}_{f_{j_{G}\left(e_{j}\right)}^{j}}^{j} ; i \neq j \text {, i.e., }<\tilde{v}_{f_{i_{G}\left(e_{i}\right)}^{i}} \widetilde{v_{f_{j_{G}\left(e_{j}\right)}}^{j}}>\tilde{=} \tilde{0} ; i \neq j .
$$

Definition 3.7 (Fuzzy soft orthonormal family). Let $\tilde{H}$ be a fuzzy soft inner product space. A family $\left\{\tilde{v}_{f_{i_{G}\left(e_{i}\right)}^{i}}^{i}\right\}$ of fuzzy soft elements of $\tilde{H}$ is called a fuzzy soft orthonormal family if $\left\{\tilde{v}_{f_{i_{G}\left(e_{i}\right)}^{i}}^{i}\right\}$ is a fuzzy soft orthogonal family (i.e., satisfies the condition (3.9)) and

$$
\left\|\widetilde{\tilde{v}_{f_{i_{G}\left(e_{i}\right)}^{i}}^{i}}\right\| \tilde{=} \tilde{1}, \forall i \text {, i.e., }<\tilde{v}_{f_{i_{G}\left(e_{i}\right)}^{i}} \widetilde{\tilde{v}_{f_{i_{G}\left(e_{i}\right)}^{i}}^{i}}>\tilde{=} \tilde{1} ; i=j .
$$

Remark 3.8. Note that if $\left\{\tilde{v}_{\mathfrak{f}_{\mathfrak{G}_{G}\left(e_{\mathfrak{i}}\right)}^{i}}\right\}$ is a fuzzy soft orthonormal family, then

$$
<\tilde{v}_{f_{i_{G}\left(e_{i}\right)}^{i}, \tilde{v}_{f_{j_{G}\left(e_{j}\right)}^{j}}^{i}}>\tilde{\delta}_{i j}
$$

where

$$
\tilde{\delta}_{i j}= \begin{cases}\tilde{1}, & \text { if } i=j, \\ \tilde{0}, & \text { if } i \neq j .\end{cases}
$$

Example 3.4. The fuzzy soft set of fuzzy soft elements $(\tilde{j}, \tilde{\theta}, \tilde{\theta}),(\tilde{\theta}, \tilde{j}, \tilde{\theta})$ and $(\tilde{\theta}, \tilde{\theta}, \tilde{j})$ is a fuzzy soft orthonormal family in $\mathbb{R}^{3}(A)$. 
Solution. Let $\tilde{e}_{1} \tilde{=}(\tilde{j}, \tilde{\theta}, \tilde{\theta}), \tilde{e}_{2} \tilde{=}(\tilde{\theta}, \tilde{j}, \tilde{\theta})$ and $\tilde{e}_{3} \tilde{=}(\tilde{\theta}, \tilde{\theta}, \tilde{j})$. It is clear that $<\widetilde{\tilde{e}_{i}, \tilde{e}_{j}}>\tilde{=} \tilde{\delta}_{i j} ; i, j=1,2,3$.

Example 3.5. The fuzzy soft set of fuzzy soft elements $(\tilde{j}, \tilde{\theta}, \tilde{\theta}, \tilde{\theta}, \ldots, \tilde{\theta}, \ldots),(\tilde{\theta}, \tilde{j}, \tilde{\theta}, \tilde{\theta}, \ldots, \tilde{\theta}, \ldots)$ and $(\tilde{\theta}, \tilde{\theta}, \tilde{j}, \tilde{\theta}, \tilde{\theta}, \ldots, \tilde{\theta}, \ldots), \ldots$ is a fuzzy soft orthonormal family in $\ell_{2}(A)$.

Solution. Let $\tilde{e}_{1} \tilde{=}(\tilde{j}, \tilde{\theta}, \tilde{\theta}, \tilde{\theta}, \ldots, \tilde{\theta}, \ldots), \tilde{e}_{2} \tilde{=}(\tilde{\theta}, \tilde{j}, \tilde{\theta}, \tilde{\theta}, \ldots, \tilde{\theta}, \ldots)$ and $\tilde{e}_{3} \tilde{=}(\tilde{\theta}, \tilde{\theta}, \tilde{j}, \tilde{\theta}, \tilde{\theta}, \ldots, \tilde{\theta}), \ldots$. It is clear that $<\widetilde{\tilde{e}_{i}, \tilde{e}_{j}}>\tilde{=} \tilde{\delta}_{i j} ; i, j=1,2,3, \ldots$

Example 3.6. Let $\tilde{\mathrm{H}} \tilde{=} \mathcal{C}_{[\tilde{0}, \tilde{1}]}(A)$ be the space of all fuzzy soft complex-valued continuous functions on $[\tilde{0}, \tilde{1}]$. For $k=0, \pm 1, \pm 2, \ldots$, let $\left\{\tilde{e}_{k}\right\} \widetilde{C} \tilde{H}$ be defined by:

$$
\tilde{e}_{k}(\tilde{x}) \tilde{=} \tilde{e}^{2 \pi i k \tilde{x}}, \tilde{x} \tilde{\in}[\tilde{0}, \tilde{1}] .
$$

Then, $\left\{\tilde{e}_{k}\right\}$ is a fuzzy soft orthonormal family in $\mathcal{C}_{[\tilde{0}, \tilde{1}]}(A)$.

\section{Solution.}

$$
\begin{aligned}
<\widetilde{\tilde{e}_{k}, \tilde{e}_{j}}>\tilde{=} \int_{\tilde{0}}^{\tilde{1}} \tilde{e}_{k}(\tilde{x}) \overline{\tilde{e}_{j}(\tilde{x})} d \tilde{x} & =\int_{\tilde{0}}^{\tilde{1}} \tilde{e}^{2 \pi i k \tilde{x}} \tilde{e}^{-2 \pi i j \tilde{x}} \mathrm{~d} \tilde{x} \\
& \simeq \int_{\tilde{0}}^{\tilde{1}} \tilde{e}^{2 \pi i(k-j) \tilde{x} d \tilde{x}} \\
& =\left\{\begin{array}{ll}
\frac{\left[\tilde{e}^{2 \pi i(k-j) \tilde{x}}\right]_{\tilde{1}}^{\tilde{1}},}{2 \pi i(k-j)}, & \text { if } k \neq j \\
\int_{\tilde{0}}^{\tilde{1}} d \tilde{x}, & \text { if } k=j
\end{array}=\left\{\begin{array}{ll}
\frac{\left(\tilde{e}^{2 \pi i(k-j)}-\tilde{1}\right)}{2 \pi i(k-j)}, & \text { if } k \neq j \\
\int_{\tilde{0}}^{\tilde{1} d \tilde{x},} & \text { if } k=j
\end{array}= \begin{cases}0, & \text { if } k \neq j \\
\tilde{1}, & \text { if } k=j .\end{cases} \right.\right.
\end{aligned}
$$

Hence, $\left\{\tilde{e}_{k}\right\}$, where $\left\{\tilde{e}_{k}\right\}$ is defined as in (3.12), is a fuzzy soft orthonormal family in $\mathcal{C}_{[\tilde{0}, \tilde{1}]}(A)$.

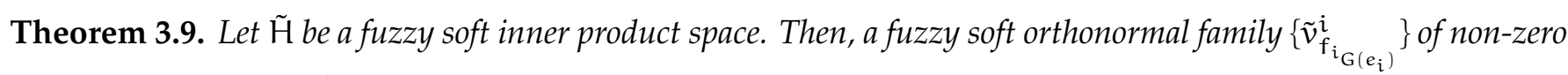
fuzzy soft elements of $\tilde{\mathrm{H}}$ is fuzzy soft linearly independent.

Proof. An arbitrary fuzzy soft set of fuzzy soft elements is called fuzzy soft linearly independent if and only if every finite fuzzy soft subset is fuzzy soft linearly independent. Let $\tilde{v}_{f_{1_{G}\left(e_{1}\right)}}^{1}, \tilde{v}_{f_{f_{G}\left(e_{2}\right)}^{2}}^{2}, \ldots, \tilde{v}_{f_{n_{G}\left(e_{n}\right)}^{n}}^{n}$ be a finite fuzzy soft subset of the fuzzy soft orthonormal family $\left\{\tilde{v}_{f_{i_{G}\left(e_{i}\right)}^{i}}\right\}$. Suppose that

$$
\tilde{\alpha}_{1} \tilde{v}_{f_{1_{G}\left(e_{1}\right)}^{1}}^{1}+\tilde{\alpha}_{2} \tilde{v}_{f_{\mathrm{G}\left(e_{2}\right)}}^{2}+\cdots+\tilde{\alpha}_{n} \tilde{v}_{f_{n_{G}\left(e_{n}\right)}^{n}}^{n} \tilde{=} \tilde{\theta}
$$

where $\tilde{\alpha}_{i}, i=1,2, \ldots, n$ are fuzzy soft scalars. By using this condition (3.13) and the Property (3) in Theorem 2.16, we obtain:

$$
\tilde{0} \tilde{=}<\tilde{\alpha}_{1} \tilde{v}_{f_{1_{G}\left(e_{1}\right)}^{1}}^{1}+\tilde{\alpha}_{2} \tilde{v}_{f_{2_{G}\left(e_{2}\right)}^{2}} \widetilde{+\cdots}+\tilde{\alpha}_{n} \tilde{v}_{f_{n_{G}\left(e_{n}\right)}^{n}}^{n}, \tilde{v}_{f_{i_{G}\left(e_{i}\right)}^{i}}^{i}>\tilde{=} \tilde{\alpha}_{i}<\tilde{v}_{f_{i_{G}\left(e_{i}\right)}^{i}} \widetilde{\nu_{f_{i_{G}\left(e_{i}\right)}^{i}}^{i}}>,
$$

for any $i=1,2, \ldots, n$. Since $<\tilde{v}_{f_{i_{G}\left(e_{i}\right)}^{i}}^{i} \widetilde{\tilde{v}_{f_{i_{G}\left(e_{i}\right)}^{i}}^{i}}>\tilde{=} \tilde{1}$ (non-zero fuzzy soft elements) for $i=1,2, \ldots, n$, then $\tilde{\alpha}_{i}=\tilde{0}, i=1,2, \ldots, n$ and hence the family $\left\{\tilde{v}_{f_{i_{G}\left(e_{i}\right)}^{i}}^{i}\right\}$ is fuzzy soft linearly independent.

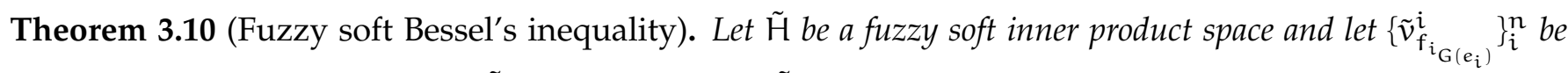
a fuzzy soft orthonormal family in $\tilde{\mathrm{H}}$. Then, for $\tilde{w}_{\mathrm{f}_{\mathrm{G}(e)}} \tilde{\in} \tilde{\mathrm{H}}$, we have:

$$
\sum_{i=1}^{n}\left|<\tilde{w}_{f_{G(e)}, \tilde{v}_{f_{i_{G}\left(e_{i}\right)}^{i}}}>\right|^{2} \tilde{\leqslant}\left\|\widetilde{\tilde{w}_{f_{G}(e)}}\right\|^{2} .
$$


Proof. Let

$$
\tilde{\mathrm{u}}_{\mathrm{g}_{\mathrm{G}(\mathrm{a})}} \tilde{=} \tilde{w}_{\mathrm{f}_{\mathrm{G}(e)}}-\sum_{i=1}^{n}<\tilde{w}_{f_{\mathrm{G}(e)}, \tilde{v}_{\mathrm{f}_{\mathrm{G}\left(e_{i}\right)}^{i}}^{i}}>\tilde{v}_{\mathrm{f}_{\mathrm{G}_{\mathrm{G}\left(e_{i}\right)}}^{i}} .
$$

Then, by taking the fuzzy soft inner product of (3.15) with $\tilde{v}_{f_{j_{G}\left(e_{j}\right)}^{j}}^{j}$ for $1 \leqslant j \leqslant n$ from the right, we get:

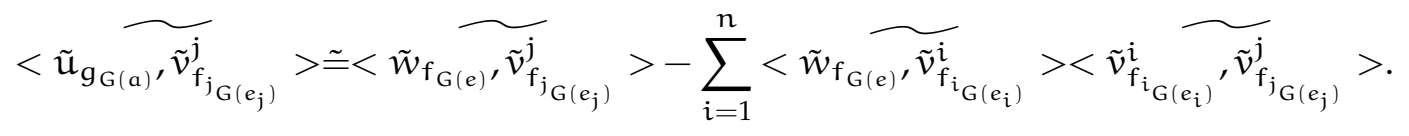

Therefore, by using Definition 3.7 of fuzzy soft orthonormal family with the related Remark 3.8 contains the equation (3.10) and its values defined by Kronecker delta (3.11) in (3.16), we obtain:

$$
\begin{aligned}
& <\tilde{u}_{g_{G(a)}, \tilde{v}_{f_{j_{G}\left(e_{j}\right)}^{j}}}>\tilde{=}<\tilde{w}_{f_{G(e)}, \tilde{v}_{f_{j_{G}\left(e_{j}\right)}^{j}}^{j}}>-<\tilde{w}_{f_{G(e)}, \tilde{v}_{f_{j_{G}\left(e_{j}\right)}^{j}}^{j}}><\tilde{v}_{f_{j_{G}\left(e_{j}\right)}^{j}} \widetilde{v_{f_{j_{G}\left(e_{j}\right)}^{j}}^{j}}> \\
& \tilde{=}<\tilde{w}_{f_{\mathrm{G}(e)}, \tilde{v}_{f_{j_{G}\left(e_{j}\right)}^{j}}^{j}}>-<\tilde{w}_{f_{G(e)}, \tilde{v}_{f_{j_{G}\left(e_{j}\right)}^{j}}}>\tilde{=} \tilde{0} \text {. }
\end{aligned}
$$

Then, $\tilde{u}_{g_{G(a)}} \tilde{\perp}_{f_{j_{G}\left(e_{j}\right)}^{j}}^{j}$ for $j=1,2, \ldots, n$. That is to say that, $\tilde{u}_{g_{G(a)}} \tilde{\perp} \tilde{\alpha}_{j} \tilde{v}_{f_{j_{G}\left(e_{j}\right)}^{j}}$ for $j=1,2, \ldots, n$ as $<\tilde{u}_{g_{G(a)}, \tilde{\alpha}_{j} \tilde{v}_{f_{j_{G}\left(e_{j}\right)}^{j}}^{j}}>\tilde{=} \tilde{\alpha}_{j}<\tilde{u}_{g_{G(a)}, \tilde{v}_{f_{j_{G}\left(e_{j}\right)}^{j}}}>\tilde{=0}$. Thus

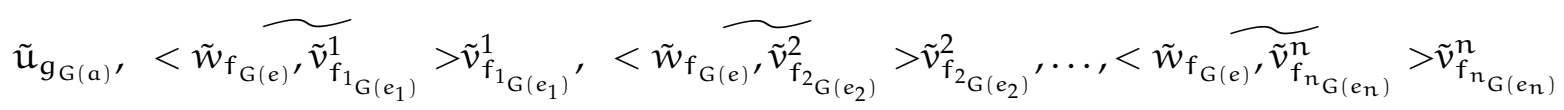

is a fuzzy soft orthonormal family, i.e,

$$
\tilde{u}_{g_{G(a)}} \tilde{I}<\tilde{w}_{f_{G(e)},} \tilde{v}_{f_{i_{G}\left(e_{i}\right)}^{i}}^{i}>\tilde{v}_{f_{i_{G}\left(e_{i}\right)}^{i}}^{i} i=1,2, \ldots, n
$$

We have:

$$
\left\|\widetilde{\tilde{w}_{f_{G}(e)}}\right\|^{2} \tilde{=}\left\|\tilde{u}_{g_{G(a)}}+\sum_{i=1}^{n}<\widetilde{\widetilde{w_{f_{G}(e)}}, \tilde{v}_{f_{i_{G}\left(e_{i}\right)}^{i}}^{i}}>\tilde{v}_{f_{i_{G}\left(e_{i}\right)}^{i}}\right\|^{2}
$$

Therefore, by applying Theorem 2.23 on (3.18) as we have (3.17), the following is obtained:

$$
\begin{aligned}
& \left\|\widetilde{\tilde{w}_{f_{G}(e)}}\right\|^{2} \tilde{=}\left\|\widetilde{\tilde{u}_{g_{G(a)}}}\right\|^{2}+\left\|\sum_{i=1}^{n}<\tilde{w}_{f_{G(e)}, \tilde{v}_{f_{i_{G}\left(e_{i}\right)}^{i}}^{i}}>\tilde{v}_{f_{i_{G}\left(e_{i}\right)}^{i}}^{i}\right\|^{2}
\end{aligned}
$$

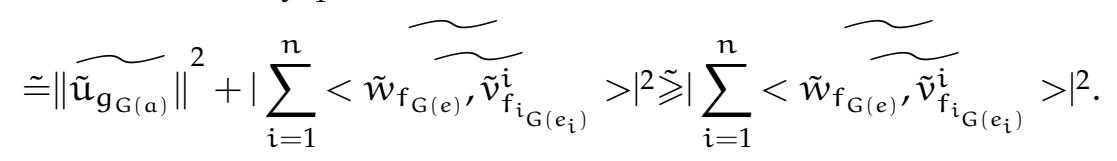

Hence, the inequality (3.14) is true and is called the Fuzzy soft Bessel's inequality.

Definition 3.11. A fuzzy soft orthonormal family $\left\{\tilde{v}_{f_{i_{G}\left(e_{i}\right)}^{i}}^{i}\right\}_{i}^{n}$, denoted by $\tilde{S}$, in a fuzzy soft inner product space $\tilde{H}$ is said to be fuzzy soft complete if there is no other fuzzy soft orthonormal family containing $\tilde{S}$ properly. That is to say that, $\tilde{S}$ must be a fuzzy soft maximal orthonormal family to be fuzzy soft complete.

Remark 3.12. A fuzzy soft orthonormal family $\tilde{S}$ is fuzzy soft complete if and only if for any $\tilde{w}_{f_{\mathrm{G}(e)}}$ such that $\tilde{w}_{f_{G(e)}} \tilde{\perp} \tilde{S}, \tilde{w}_{f_{G}(e)}$ must be the fuzzy soft zero vector $\tilde{\theta}$. 
Theorem 3.13. Suppose that $\tilde{\mathrm{H}}$ is a fuzzy soft Hilbert space and let $\left\{\tilde{v}_{\mathrm{f}_{\mathrm{G}_{\mathrm{G}}\left(e_{i}\right)}^{\mathrm{i}}}\right\}$ be a fuzzy soft countably infinite orthonormal family in $\tilde{\mathrm{H}}$. Then, a fuzzy soft series of the form

$$
\sum_{i=1}^{\infty} \tilde{\lambda}_{i} \tilde{v}_{f_{i_{G}\left(e_{i}\right)}^{i}}^{i} \text { is fuzzy soft convergent } \Leftrightarrow \sum_{i=1}^{\infty}\left|\tilde{\lambda}_{i}\right|^{2} \tilde{<} \infty,
$$

for fuzzy soft scalar $\tilde{\lambda}_{i}$, and in this case, we have the following relations:

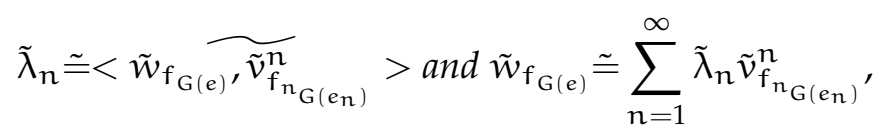

between the fuzzy soft coefficients $\tilde{\lambda}_{n}$ and the fuzzy soft element $\tilde{w}_{\mathrm{f}_{\mathrm{G}(e)}}$ defined by the fuzzy soft series.

Proof. First, to prove (3.19), let

$$
\tilde{S}_{\mathrm{n}} \cong \sum_{i=1}^{n} \tilde{\lambda}_{n} \tilde{v}_{\mathrm{f}_{\mathrm{G}_{\mathrm{G}\left(e_{n}\right)}}^{n}}
$$

Then, if $m<n$, we have:

$$
\begin{aligned}
& \left\|\tilde{S}_{n}-\tilde{S}_{m}\right\|^{2} \tilde{=}\left\|\sum_{i=1}^{n} \tilde{\lambda}_{i} \tilde{v}_{f_{i_{G}\left(e_{i}\right)}^{i}}-\sum_{i=1}^{m} \tilde{\lambda}_{i} \tilde{v}_{f_{i_{G}\left(e_{i}\right)}^{i}}\right\|^{2} \\
& \tilde{=}\left\|\sum_{i=m+1}^{n} \widetilde{\tilde{\lambda}_{i} \tilde{v}_{f^{i}\left(e_{i}\right)}^{i}}\right\|^{2} \tilde{\leqslant} \sum_{i=m+1}^{n}\left\|\tilde{\lambda}_{i} \widetilde{\tilde{v}_{f_{i_{G}\left(e_{i}\right)}^{i}}^{i}}\right\|^{2} \tilde{=} \sum_{i=m+1}^{n}\left|\tilde{\lambda}_{i}\right|^{2}\left\|\widetilde{v_{f_{i_{G}\left(e_{i}\right)}}^{i}}\right\|^{2} .
\end{aligned}
$$

Using the given that $\left\{\tilde{v}_{f_{i_{G}\left(e_{i}\right)}^{i}}^{i}\right\}$ is a fuzzy soft orthonormal family in $\tilde{H}$ (i.e., $\left.\left\|\widetilde{\tilde{v}_{f_{G}\left(e_{i}\right)}^{i}}\right\|^{2} \tilde{=} \tilde{1}\right)$ and substituting in (3.22), we get:

$$
\left\|\tilde{S}_{n}-\tilde{S}_{m}\right\|^{2} \tilde{\leqslant} \sum_{i=m+1}^{n}\left|\tilde{\lambda}_{i}\right|^{2} \tilde{<} \infty .
$$

Then, from (3.23), we obtain: $\left\{\tilde{S}_{n}\right\}_{1}^{\infty}$ is fuzzy soft Cauchy sequence $\Leftrightarrow \sum_{i=1}^{\infty}\left|\tilde{\lambda}_{i}\right|^{2} \tilde{<} \infty$. Since $\tilde{H}$ is fuzzy soft Hilbert space, i.e., it is fuzzy soft complete. Then, $\left\{\tilde{S}_{\mathrm{n}}\right\}_{1}^{\infty}$ is fuzzy soft convergent sequence $\Leftrightarrow$ $\sum_{i=1}^{\infty}\left|\tilde{\lambda}_{i}\right|^{2} \tilde{<} \infty$. Now to prove the two formulas (3.20), use (3.21) and let

$$
\tilde{w}_{f_{G(e)}} \tilde{=} \lim _{n \rightarrow \infty} \sum_{i=1}^{n} \tilde{S}_{i} \tilde{=} \lim _{n \rightarrow \infty} \tilde{S}_{n} \tilde{=} \sum_{i=1}^{\infty} \tilde{S}_{i} \tilde{=} \sum_{i=1}^{\infty} \tilde{\lambda}_{i} \tilde{v}_{f_{i_{G}\left(e_{i}\right)}^{i}} .
$$

Therefore, using (3.21), applying the properties which are stated in Theorem 2.16 and with the help of the given that $\left\{\tilde{v}_{f_{i_{G}\left(e_{i}\right)}^{i}}\right\}$ is a fuzzy soft orthonormal family in $\tilde{H}$, we obtain, for $i=1,2, \ldots, n$ :

$$
<\tilde{S}_{n}, \widetilde{\tilde{v}_{f_{i_{G}\left(e_{i}\right)}^{i}}^{i}}>\tilde{=}<\sum_{j=1}^{n} \tilde{\lambda}_{j} \tilde{v}_{f_{j_{G}\left(e_{j}\right)}^{j}}^{j}, \tilde{v}_{f_{i_{G}\left(e_{i}\right)}^{i}}^{i}>\tilde{=} \sum_{j=1}^{n} \tilde{\lambda}_{j}<\tilde{v}_{f_{j_{G}\left(e_{j}\right)}^{j},} \widetilde{\tilde{v}_{f_{i_{G}\left(e_{i}\right)}^{i}}^{i}}>\tilde{=} \sum_{j=1}^{n} \tilde{\lambda}_{j} \tilde{\delta}_{i j} \tilde{=} \tilde{\lambda}_{i} ; \tilde{\delta}_{i j} \tilde{=1}, i=j
$$

After that, from (3.24), we have $\tilde{S}_{n} \rightarrow \tilde{w}_{f_{G(e)}}$ as $n \rightarrow \infty$, and hence by using the fuzzy soft continuity of the fuzzy soft inner product space (Theorem 2.21), we get:

$$
<\tilde{S}_{n, \tilde{v}_{f_{i_{G}\left(e_{i}\right)}^{i}}^{i}}>\rightarrow<\tilde{w}_{f_{G(e)},} \tilde{v}_{f_{i_{G}\left(e_{i}\right)}^{i}}^{i} \text { as } n \rightarrow \infty .
$$


Thus, from (3.25) and (3.26), one of the two required formulas which is $\tilde{\lambda}_{i} \tilde{=}<\tilde{w}_{f_{G(e)}, \widetilde{v_{f_{G}\left(e_{i}\right)}^{i}}}>$ is obtained and substituting it in (3.24), the second required formula is obtained as follows:

$$
\tilde{w}_{f_{G(e)}} \simeq \sum_{i=1}^{\infty}<\tilde{w}_{f_{G(e)}, \tilde{v}_{f_{i_{G}\left(e_{i}\right)}^{i}}^{i}}>\tilde{v}_{f_{i_{G}\left(e_{i}\right)}^{i}} .
$$

Theorem 3.14 (Fuzzy soft Parseval's formula). Let $\tilde{S}$ be a fuzzy soft orthonormal family in a fuzzy soft inner product space $\tilde{\mathrm{H}}$, and suppose that

$$
\left\|\widetilde{\tilde{w}_{f_{\mathrm{G}(e)}}}\right\|^{2} \tilde{=} \sum_{\tilde{v}_{f_{\mathrm{G}(e)}} \tilde{\epsilon} \tilde{\mathrm{S}}}\left|<\tilde{w}_{f_{\mathrm{G}(e)}, \tilde{v}_{\mathrm{f}_{\mathrm{G}(e)}}}>\right|^{2} \text { for every } \tilde{w}_{\mathrm{f}_{\mathrm{G}(e)}} \tilde{\epsilon} \tilde{\mathrm{H}} .
$$

Then, $\tilde{\mathrm{S}}$ is fuzzy soft complete.

On the other hand, if $\tilde{\mathrm{H}}$ is a fuzzy soft Hilbert space (fuzzy soft complete) and $\tilde{\mathrm{S}}$ is a fuzzy soft complete orthonormal family in $\tilde{\mathrm{H}}$, then the equality holds for every $\tilde{w}_{\mathrm{f}_{\mathrm{G}(e)}} \tilde{\in} \tilde{\mathrm{H}}$. The Formula (3.27) is called fuzzy soft Parseval's formula.

Proof. To prove the first part (the necessary condition), suppose that $\tilde{S}$ is not fuzzy soft complete, then there exists some $\tilde{w}_{f_{\mathrm{G}(e)}} \tilde{=} \tilde{\theta}$ such that $\tilde{w}_{f_{\mathrm{G}(e)}} \tilde{\perp} \tilde{S}$. But (3.27) gives $\left\|\widetilde{\tilde{w}_{f_{\mathrm{G}(e)}}}\right\|^{2} \tilde{=0}$, and then $\tilde{w}_{f_{\mathrm{G}(e)}} \tilde{=} \tilde{\theta}_{\text {, which is }}$ contradiction. Hence, $\tilde{S}$ is fuzzy soft complete.

To prove the second part (the sufficient condition), suppose that $\tilde{H}$ is a fuzzy soft complete inner product space (fuzzy soft Hilbert space) and $\tilde{S}$ is a fuzzy soft complete orthonormal family in $\tilde{H}$. Then, by using Theorem 3.13, we have for every $\tilde{w}_{f_{G}(e)} \tilde{\in} \tilde{H}$, there is some finite or countably infinite fuzzy soft family $\left\{\tilde{v}_{f_{n_{G}\left(e_{n}\right)}^{n}}\right\}$ in $\tilde{S}$ such that the fuzzy soft series of the form $\sum_{i=1}^{\infty} \tilde{\lambda}_{i} \tilde{v}_{f_{i_{G}\left(e_{i}\right)}^{i}}$ is fuzzy soft convergent if and only

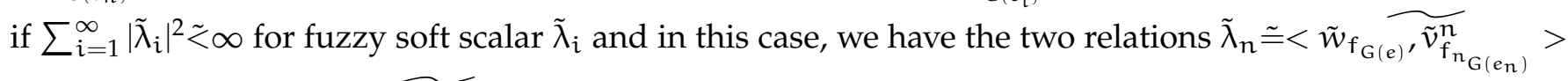
and $\tilde{w}_{f_{\mathrm{G}(e)}} \tilde{=} \sum_{n=1}^{\infty}<\tilde{w}_{f_{\mathrm{G}(e)}, \tilde{v}_{f_{n_{G}\left(e_{n}\right)}^{n}}^{n}}>\tilde{v}_{f_{n_{G}\left(e_{n}\right)}^{n}}$ between the fuzzy soft coefficients $\tilde{\lambda}_{n}$ and the fuzzy soft element $\tilde{w}_{f_{G(e)}}$ defined by the fuzzy soft series. That is to say that,

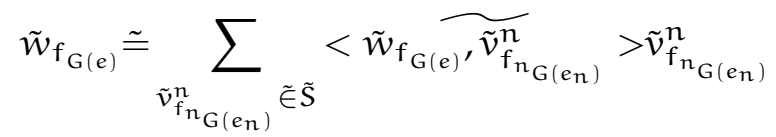

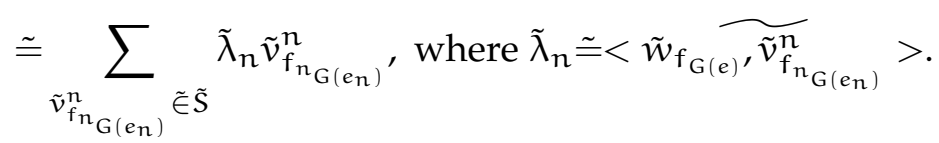

By applying Theorem 2.19, substituting for $\tilde{w}_{f_{G}(e)}$ from (3.28), with the help of the properties stated in Theorem 2.16 and using the given that $\tilde{S}$ is a fuzzy soft orthonormal family in $\tilde{H}$, we obtain:

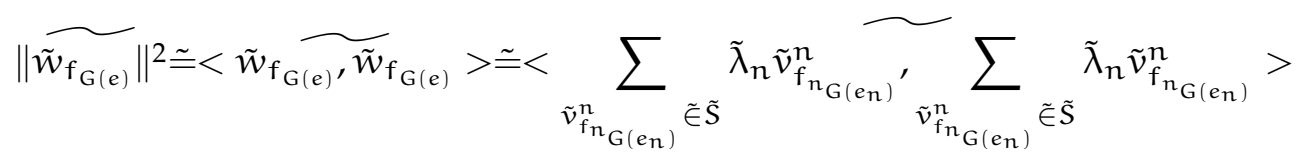

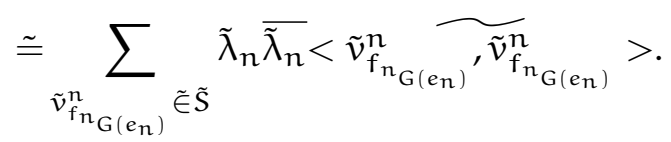

Since, $\tilde{\lambda}_{n} \tilde{\lambda}_{n} \tilde{=}\left|\tilde{\lambda}_{n}\right|^{2}$, then we have:

$$
\left\|\widetilde{\tilde{w}_{f_{G}(e)}}\right\|^{2} \cong \sum_{\tilde{v}_{f_{n_{G}\left(e_{n}\right)}^{n}}^{n}}\left|\tilde{\lambda}_{n}\right|^{2}\left\|\tilde{v}_{f_{n_{G}\left(e_{n}\right)}^{n}}^{\sim}\right\|^{2} \tilde{=} \sum_{\tilde{v}_{f_{n_{G}\left(e_{n}\right)}^{n}}^{n}}\left|\tilde{\lambda}_{n}\right|^{2} .
$$


Now, by substituting for $\lambda_{n}$ from (3.28) in (3.29), we get:

$$
\left\|\widetilde{\tilde{w}_{f_{G}(e)}}\right\|^{2} \tilde{=} \sum_{\tilde{v}_{f_{n_{G}\left(e_{n}\right)}^{n}}^{n}}\left|<\tilde{w}_{f_{G}(e),} \widetilde{\tilde{v}_{f_{n_{G}\left(e_{n}\right)}^{n}}^{n}}>\right|^{2} .
$$

That is to say that,

$$
\widetilde{\| \tilde{w}_{f_{G}(e)}} \|^{2} \tilde{=} \sum_{\tilde{v}_{f_{G}(e)} \tilde{\in} \tilde{S}}\left|<\tilde{w}_{f_{G(e)}, \tilde{v}_{f_{G}(e)}}>\right|^{2}
$$

for every $\tilde{\mathfrak{w}}_{\mathrm{f}_{\mathrm{G}(e)}} \tilde{\in} \tilde{\mathrm{H}}$.

Corollary 3.15. If $\tilde{\mathrm{H}}$ is a fuzzy soft Hilbert space and $\tilde{\mathrm{S}}$ is a fuzzy soft complete orthonormal family in $\tilde{\mathrm{H}}$, then for $\tilde{w}_{f_{1_{\mathrm{G}}\left(e_{1}\right)}^{1}}^{1}, \tilde{w}_{\mathrm{f}_{2_{\mathrm{G}}\left(e_{2}\right)}}^{2} \tilde{\in} \tilde{\mathrm{H}}$, we have:

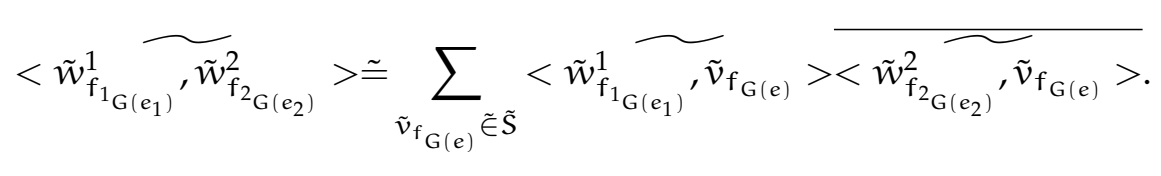

\section{Conclusions and future work}

On the one hand, the fuzzy version or the soft version of topics like metric spaces, normed spaces, Banach spaces, operators, dual spaces, functionals, inner product spaces, Hilbert spaces, operators on Hilbert spaces and many other topics have been studied by many mathematicians. On the other hand, combining fuzzy and soft sets together is not only more general than using only one of them, but also gives us more extended and accurate results. Few researchers have studied some of those general extensions concepts such as fuzzy soft normed spaces and fuzzy soft metric spaces. We have defined the fuzzy soft Hilbert space $\tilde{H}$ by using the concept of fuzzy soft vector $\tilde{v}_{f_{G(e)}}$ modified in our work and have shown that $\mathbb{C}^{\mathfrak{n}}(A), \mathbb{R}^{\mathfrak{n}}(A)$ and $\ell_{2}(A)$ are suitable examples of fuzzy soft Hilbert spaces. Moreover, some related properties of fuzzy soft Hilbert spaces have been introduced. In addition, we have defined the fuzzy soft orthogonal family and the fuzzy soft orthonormal family. Furthermore, we have established fuzzy soft Bessel's inequality and fuzzy soft Parseval's formula. Our work can be considered as a generalization for the well-known (crisp) Hilbert spaces. This type of investigation fills some gaps in the literature. The authors can introduce new results by using similar techniques in this paper. Some fuzzy soft linear operators in fuzzy soft Hilbert spaces will be studied in further investigations depending on the definition of fuzzy soft Hilbert space given in this paper. Finally, there are still many topics to study by applying the fuzzy soft notion on them.

\section{Acknowledgment}

The authors would like to thank the referees for their valuable suggestions and comments.

\section{References}

[1] S. Bayramov, C. Gunduz, Soft locally compact spaces and soft paracompact spaces, J. Math. Sys. Sci., 3 (2013), $122-130$. 1

[2] T. Beaula, C. Gunaseeli, On fuzzy soft metric spaces, Malaya J. Mat., 2 (2014), 197-202. 1, 2.7

[3] T. Beaula, M. M. Priyanga, A new notion for fuzzy soft normed linear space, Int. J. Fuzzy Math. Arch., 9 (2015), 81-90. 1, 2.6, 2.8

[4] T. Beaula, M. M. Priyanga, Fuzzy soft linear operator on fuzzy soft normed linear spaces, Int. J. App. Fuzzy Sets Art. Intell., 6 (2020), 73-92. 2.8

[5] S. Das, S. K. Samanta, On soft inner product spaces, Ann. Fuzzy Math. Inform., 6 (2013), 151-170. 1

[6] S. Das, S. K. Samanta, Soft Metric, Ann. Fuzzy Math. Inform., 6 (2013), 77-94. 1 
[7] N. Faried, M. S. S. Ali, H. H. Sakr, Fuzzy soft inner product spaces, Appl. Math. Inf. Sci., 14 (2020), 709-720. 1, 2.15, $2.16,2.17,2.18,2.19,2.20,2.21,2.22,2.23,2.24,2.25$

[8] C. Gunaseeli, Some Contributions to Special Fuzzy Topological Spaces, Ph.D. Thesis (Bharathidasan University), India, (2012). 1

[9] K. Hayat, M. I. Ali, J. C. R. Alcantud, B.-Y. Cao, K. U. Tariq, Best concept selection in design process: An application of generalized intuitionistic fuzzy soft sets, J. Intell. Fuzzy Syst., 35 (2018), 5707-5720. 1

[10] A. Z. Khameneh, A. Kiliçman, A. R. Salleh, Parameterized norm and parameterized fixed-point theorem by using fuzzy soft set theory, arXiv, 2013 (2013), 15 pages. 2.9, 2.10, 2.11, 2.12, 2.13, 2.14

[11] J. Mahanta, P. K. Das, Fuzzy soft topological spaces, J. Intell. Fuzzy Syst., 32 (2017), 443-450. 2.5

[12] T. Mahmood, M. I. Ali, M. A. Malik, W. Ahmed, On lattice ordered intuitionistic fuzzy soft sets, Int. J. Algebra Stat., 7 (2018), 46-61. 1

[13] P. K. Maji, R. Biswas, A. R. Roy, Intuitionistic fuzzy soft sets, J. Fuzzy Math., 9 (2001), 677-692. 1, 2.3

[14] P. K. Maji, R. Biswas, A. R. Roy, Soft set theory, Comput. Math. Appl., 45 (2003), 555-562. 2.2

[15] D. Molodtsov, Soft set theory-first results, Comput. Math. Appl., 37 (1999), 19-31. 1, 2.2

[16] T. J. Neog, D. K. Sut, G. C. Hazarika, Fuzzy soft topological spaces, Int. J. Latest Trend Math., 2 (2012), 54-67. 1, 2.4

[17] N. Sultana, N. Rani, M. I. Ali, A. Hussain, Soft translations and soft extensions of BCI/BCK-algebras, Sci. World J., 2014 (2014), 6 pages. 1

[18] M. I. Yazar, C. G. Aras, S. Bayramov, Results on soft Hilbert spaces, TWMS J. App. Eng. Math., 9 (2019), 159-164. 1

[19] M. I. Yazar, T. Bilgin, S. Bayramov, C. Gunduz, A new view on soft normed spaces, Int. Math. For., 9 (2014), 1149-1159. 1

[20] L. A. Zadeh, Fuzzy set, Information and Control, 8 (1965), 338-353. 1, 2.1 